\title{
Deep Learning Algorithms based Fingerprint Authentication: Systematic Literature Review
}

\author{
Haruna Chiroma \\ University of Hafr Al-Batrin, College of Computer Science and Engineering, Hafr Al-Batin, Saudi Arabia \\ Email: freedonchi@yahoo.com
}

How to cite this paper: Haruna Chiroma (2021). Deep Learning Algorithms based Fingerprint Authentication: Systematic Literature Review. Journal of Artificial Intelligence and Systems, 3, 157-197. https://doi.org/10.33969/AIS.2021.31010

Received: October 19, 2021

Accepted: November 22, 2021

Published: December 10, 2021

Copyright (C) 2021 by author(s) and Institute of Electronics and Computer. This work is licensed under the Creative Commons Attribution International License (CC BY 4.0).

http://creativecommons.org/licenses/by/4.0/

\begin{abstract}
Deep Learning algorithms (DL) have been applied in different domains such as computer vision, image detection, robotics and speech processing, in most cases, DL demonstrated better performance than the conventional machine learning algorithms (shallow algorithms). The artificial intelligence research community has leveraged the robustness of the DL because of their ability to process large data size and handle variations in biometric data such as aging or expression problem. Particularly, DL research in automatic fingerprint recognition system (AFRS) is gaining momentum starting from the last decade in the area of fingerprint pre-processing, fingerprints quality enhancement, fingerprint feature extraction, security of fingerprint and performance improvement of AFRS. However, there are limited studies that address the application of DL to model fingerprint biometric for different tasks in the fingerprint recognition process. To bridge this gap, this paper presents a systematic literature review and an insightful meta-data analysis of a decade applications of DL in AFRS. Discussion on proposed model's tasks, state of the art study, dataset, and training architecture are presented. The Convolutional Neural Networks models were the most saturated models in developing fingerprint biometrics authentication. The study revealed different roles of the DL in training architecture of the models: feature extractor, classifier and end-to-end learning. The review highlights open research challenges and present new perspective for solving the challenges in the future. The author believed that this paper will guide researchers in propose novel fingerprint authentication scheme.
\end{abstract}

Keywords

Biometrics, Fingerprints, Machine Learning, Deep Learning, Fingerprint Analysis

\section{Introduction}

Deep learning methods (DL) are subset of machine learning algorithms, which are derived from artificial neural networks. Unlike the conventional machine learning algorithms (shallow algorithms) that use single hidden layer with input and output 
layers, DL involve the use of at least two hidden layers. The term "deep" in DL connotes the idea of hierarchical layers of representations, with modern DL having several hierarchical layers of representations for learning features [1, 2]. Based on the literature, DL have produced superior performance against the conventional machine learning methods or traditional approaches. The popularity and success of DL over the traditional approaches can be associated to factors such as the ability of to learn features automatically unlike hand-engineering feature selection methods, the deep hierarchical representations and the ability for distribution of representation [3]. More so, the introduction of powerful processor hardware such as graphic processing units has enhanced the processing power of the large-scale datasets. This further helped in demonstrating the robustness of data intensive DL [4].

The DL methods have been widely used in cybersecurity science. For example, the DL has being found to be applied in modeling biometrics in forensic science for criminal investigations [5] and digital security applications for authentication of individuals [6]. The applications of DL in biometrics recognition and authentication has also received attention in recent years, with various schemes developed to improve digital security and safety issues [7]. Biometrics refers to the measurement of physiological and behavioral features for recognizing individuals automatically [8]. Biometrics is a better alternative to knowledge-based recognition (the use of password) systems. Biometrics systems have been used to identify and assign privileges to individuals based on their physiological and behavioral characteristics.

These characteristics have been identified as unique and consistent to individuals. Even the fingerprint patterns in twins have been investigated to be unique and in 1893, it was formerly accepted by the Home Ministry Office, UK that no two humans possess the same fingerprints [9]. These unique features have made biometric systems difficult to be by-passed by spoofing attacks. Biometric systems can be based on physiological characteristics such as fingerprint [10], iris [11], and hand veins [12]. Secondly, behavioral traits, such as keystroke dynamics [13]. Thirdly, signature [14]. As reported in the literature, fingerprints is considered as the most used biometric for the automatic fingerprint recognition system (AFRS) [15].

Beside biometrics, DL have witnessed tremendous applications in various domain such as computer vision, remote sensing, natural language processing, education, etc. [16]. The focus of the study is biometrics. Specifically, DL models have been utilized to model various biometrics for human recognition and authentication. Also, DL have also been used to enhance, segment and extract fingerprint features towards a faster recognition. This is due to the impacts of the DL to process large amount of data and the ability to train discriminatively and handle variations of biometric data challenge [3]. In addition, the DL have the potency to model expression or aging problem associated with biometric traits $[17,18]$. The DL models have proven to be effective, and superior compared to the state-of-the-art approaches for biometric recognition systems, especially in AFRS. According to the review conducted by [19], DL approach is observed to be heavily investigated from 2012 to 2019. The authors argued that this will remain the active direction for the fingerprint research in the future.

Fingerprint biometric is believed to be the fastest, most common, and oldest method for physiological biometric systems and very well acknowledged by the legal community [20-22]. More so, the implementation of fingerprint authentication 
system is easy, cheap, convenient and with a satisfactory level of accuracy [23-25]. In addition, fingerprint identification has been applied in almost all security protection areas and has a higher universality compared to other biometrics systems [26]. Fingerprints have widely been used in different domains including criminal investigations and in digital security systems to facilitate criminal investigations and strengthen security systems, respectively. There are researches conducted on the application of DL in biometric recognition and authentication schemes have been surveyed in [3, 27-32]. However, these previous studies mainly focused on a particular application area, such as security[29], fingerprint liveness detection[28], fingerprint classification[30], fingerprint enhancement[32] etc. or study based on biometrics with fingerprint inclusive $[3,27,31]$ and some are not DL based surveys on the contrary[28, 29,32]. In addition, those surveys were not systematic and did not present the applications of DL in fingerprint biometrics analyses based on different areas of applications. To bridge the gap, this study intents to presents a comprehensive systematic literature review of the applications of DL in fingerprint biometrics in different applications domain unlike the previous reviews. The contributions of this review paper are summarized as follows:

i. The study presents an insightful meta-data analysis of a decade applications of DL in fingerprint biometric analyses including the choice of DL framework, application tasks, model performances, datasets, and model training architecture.

ii. Unlike previous reviews, this review paper presents different areas of applications where DL were used in fingerprint biometric.

iii. The study highlighted presents challenges and potential future directions for DL-based AFRS research.

The rest of this paper is structured as follows: In section 2, theoretical frameworks of DL architectures and fingerprints biometric are presented. Section 3, presents the methodology adopted for this review paper. In section 4, applications of DL in fingerprint biometric recognition and analyses are presented. Section 5, presents discussions on the DL models, application tasks, datasets, and training architecture. The open research issues and future directions are outlined in section 6. Finally, section 7 concludes the paper.

\section{Theoretical Framework}

This section discusses the theoretical frameworks of some commonly used DL in AFRS and the general fingerprint biometric recognition and classification processes.

\subsection{Deep Learning Architectures}

DL is inspired by artificial neural networks [33, 34]. The DL models apply linear and nonlinear transformations of the input data through the "deep" layers. The DL have proven effective over state-of-the-art methods in handling many data analytic application tasks such as image classification [35], speech recognition [36, 37], object detection [38], speech and computer vision [4, 39], and biometric authentication [3]. In this section, we presented some commonly used DL frameworks for biometric (fingerprints) analyses. 
Convolutional Neural Network: The concept of CNNs was derived based on the human visual cortex [40]. The CNNs are the most utilized DL for computer vision, image detection and video recognition [41]. It is a DL-based technique designed to learn features from an input image/data and classifies the input data into the desired classes. The classical CNN structure has three building blocks, the convolution layers and the pooling layers which are normally employed to extract features and fully connected layer is used for classification [4, 42]. The convolution operation is mathematically expressed as in equation (1) [34]:

$$
x_{x j}^{1}=f\left(\sum_{i \in M j} x^{I-1} * \begin{array}{c}
I \\
x_{i j}+{ }_{b J}
\end{array}\right)
$$

$$
\begin{array}{cl}
1 & =\text { current layer output } \\
x j & =\text { previous layer output } \\
I-1 & =\text { current layer kernel } \\
x i & \\
I & =\text { bias of the current layer } \\
K i j & \\
I &
\end{array}
$$$$
\begin{array}{cl}
I & =\text { current layer kernel } \\
K_{i j} &
\end{array}
$$$$
M_{j} \quad=\text { selection of the input maps }
$$

The convolved features would pass to the pooling layer which performs sub-sampling operations and then passed to the next convolutional layer. There are different pooling operations such as max, average and sum pooling. Max and average pooling are the most popular [4, 34]. The sub-sampling operation is computed using equation (2):

$$
\frac{1}{x j}=\operatorname{down}\left(\begin{array}{c}
I-1 \\
x j
\end{array}\right)
$$

The CNNs are widely used models for time series and image data classification [43-46]. There are different variants of CNN proposed in the literature such as AlexNet [47] ResNet [48], VGGnet [49], SqueezeNet [50] and GoogLeNet[51]. The reader is referred to a more detailed discussion on DL architectures based on CNNs in [52]. Figure 1 shows the classical structure of the CNNs. 


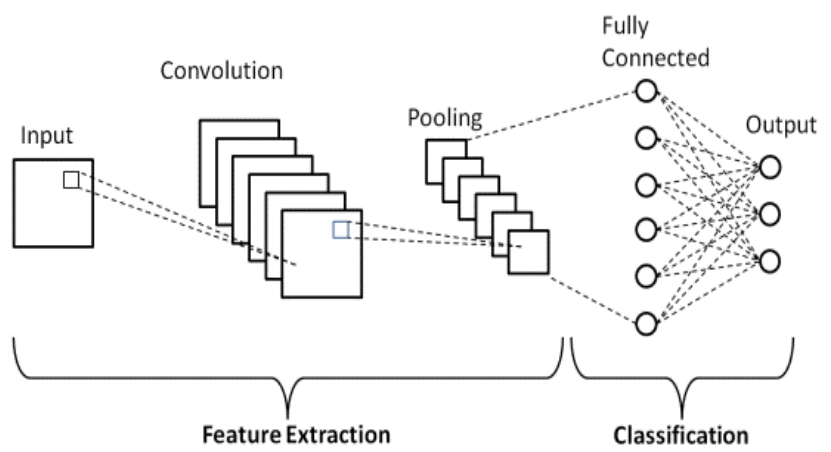

Figure 1. The classical CNN structure [53].

Restricted Boltzmann Machine: The restricted Boltzmann machine (RBM) was proposed by Paul Smolensky in 1986. However, called it Harmonium [54]. The RBM is a network of stochastic units widely used to learn the input probability distribution in unsupervised and supervised approach, hence, its popularity as DL framework [55]. Deep Belief Networks (DBN) as well as Deep Boltzmann Machines are two main DL architectures that incorporates RBM, both are considered to belong to the "Boltzmann family" [4]. In DBN, the top two layers has undirected connections and all its subsequent layers have directed connections between them [4] (see Figure 2). DBN learns to extract a deep hierarchical representation of the data under training. They model the joint distribution between observed vector $\boldsymbol{x}$ and the hidden layer as in equation (3).

$$
p\left(x,{ }_{h}^{1}, \ldots . .,{ }_{h}^{1}\right)=\left(\prod_{k=0}^{i-2} P\left(\left.h^{k}\right|_{h} ^{k+1}\right)\right) p\left(h^{i-1}, h\right)
$$

Where $x={ }_{h}^{o}, p\left(\left.{ }_{h}{ }^{k}\right|_{h}{ }^{k+1}\right)$ represents a conditional distribution for the visible units at level $k$ conditioned on the hidden units of the RBM at level $k+1$ and $p\left(h^{l-1} \mid h^{l}\right)$ is the visible-hidden joint distribution in the top-level RBM [4]. A detailed discussion on RBM and how it's used to trained data is reviewed in [56].

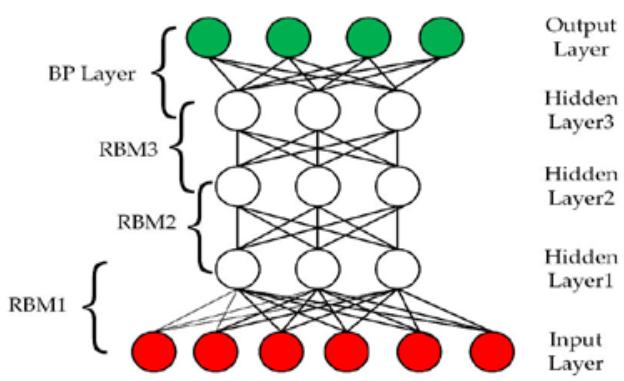

Figure 2. The structure of DBN[57] 
Autoencoder: An autoencoder (AE) is an unsupervised DL approach originally proposed by Lecun et al., in 1987 [58]. It involves dimensional reduction of the input data and reconstruction of the input in the output layer [41] (see Figure 3). Training in AE technique involves two phases: The encoder and the decoder. The network is trained using backpropagation algorithm. During the encoding phase, the inputs are encoded into some hidden representations (using weight metrics of the lower half layer) and it tries to reconstruct the same input from the encoding representation in the decoding phase using the metrics of the upper half layer. The encoding and decoding phases can be mathematically expressed as in equation (4) and (5) respectively:

$$
\begin{aligned}
& x=f(w x+b) \\
& x^{\prime}=f\left(\begin{array}{c}
\prime \\
w y+c
\end{array}\right)
\end{aligned}
$$

Where $x$ and $x^{\prime}$ represents the input vector and reconstructed input vector in the output layer respectively. Variable $\mathrm{w}$ and $b$ are the parameters to be turned, $\boldsymbol{w}^{\prime}$ and $c$ is the transpose of $w$, and the bias of the output layer respectively; $y$ is the hidden representation and $f$ is the activation function. The parameters are updated using the following equations:

Where $\boldsymbol{w}_{\text {new }}$ and $\boldsymbol{b}_{\text {new }}$ stands for the updated parameters of $\mathrm{w}$ and $b$ respectively and $\boldsymbol{E}$ is the reconstructed error of input at the output layer [55]. Discussion on AE and its different variant in used today can be found in [59]

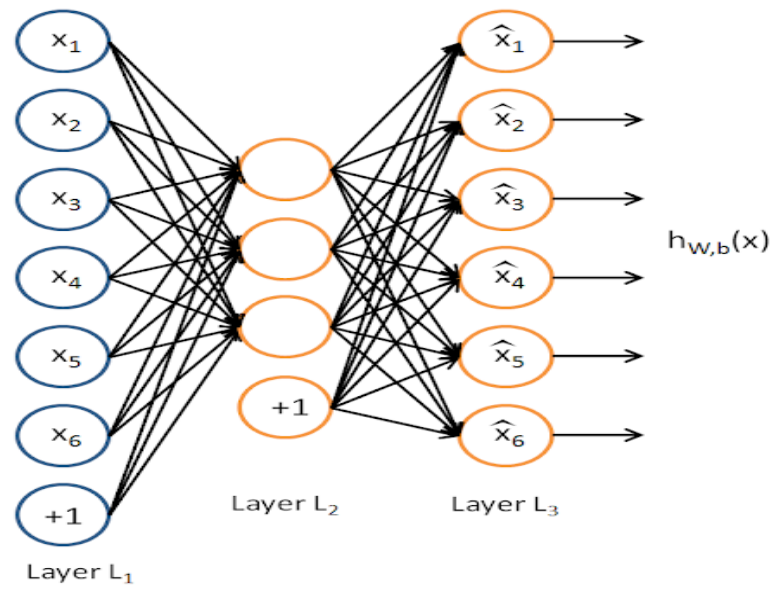

Figure 3. The classical AE Structure

Generative adversarial network: The generative adversarial network (GAN) is a DL algorithm with unsupervised learning approach proposed by Goodfellow et al. in 2014 [60]. The GAN have two networks, namely, generative and discriminator networks that compete in such a way that one gains while the other losses [34]. The GANs could discover and learn the patterns during the training of fingerprint in such a way that they can generate a new set of data like the input data. During the training of data, the generator network trains to generate new examples while the discriminator network classifies examples as either real or fake [61]. The zero-sum 
game between the two networks goes on until the generator network can generate plausible examples. The GAN has been used to generate replica of image data in a process called data augmentation. A detail discussion on GANs and its variants is reviewed in [62]. Figure 4 shows the GAN structural representation [61].

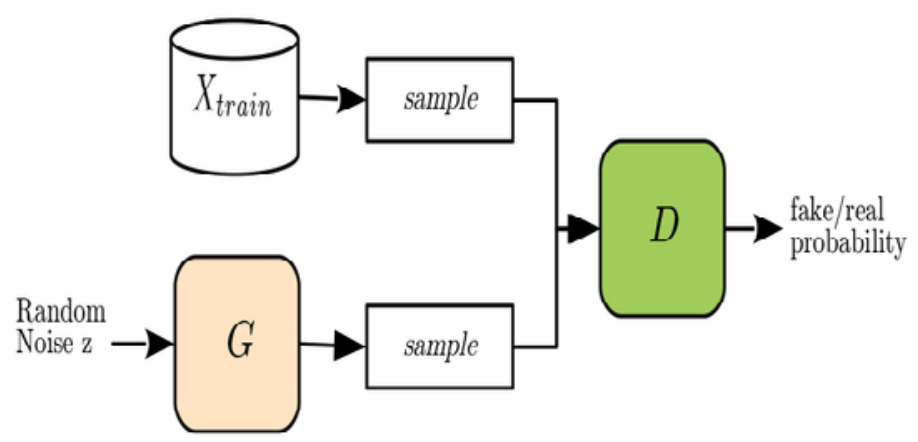

Figure 4. The GAN structure representation

\subsection{Fingerprint Biometrics}

Fingerprint biometric is one of the most utilized physiological biometrics found in humans. The fingerprint is the pattern formed or impression made when a human finger meets an object. This impression made is a representation of the epidermis (ridge lines) and dermis (spaces in-between ridges) of a finger [21]. The fingerprint as a biometric characteristic has been used for so many applications and for various reasons. The use of fingerprint for human authentication and identification has become commonplace because of its uniqueness and easy implementation [24, 25]. The characteristics of fingerprint biometric have been found to possess some uniqueness and consistency (permanence) in humans that uniquely identify everyone and can prevent fraud, enhance security and curtail identity theft [7]. Fingerprint biometric systems have been applied in different area of applications ranging from forensic investigation, customs access, law enforcement, public security organizations and healthcare environment [5, 63].

The classification of fingerprint has been studied since from its discovery as a unique human characteristic to identify individuals. The anatomy of fingerprint can be viewed through the pattern formed or the impression of the fingerprint pattern formed on touched object. The ridge (also called epidermis) is denoted as black lines while the valleys (also called dermis) refer to the white spaces between the ridges (see Figure 5)[21]. Minutiae are the vital components during fingerprint recognition. It refers to the point where the ridge structure changes [22, 64]. On the average, each fingerprint has been estimated to have about 70 minutiae and the common ones are termination, bifurcation, lake, independent ridge, point or island, spur and crossover [21]. Based on the ridge pattern, five classes of fingerprint can be observed, this is called Henry's fingerprint classification (Figure 6)[65, 66]. As shown in the figure, the various categories include Arch, left loop, right loop, tented arch, and whorl. However, arch is normally classified in place of arch and tended arch in the real AFRS because of their low occurrence [67]. 


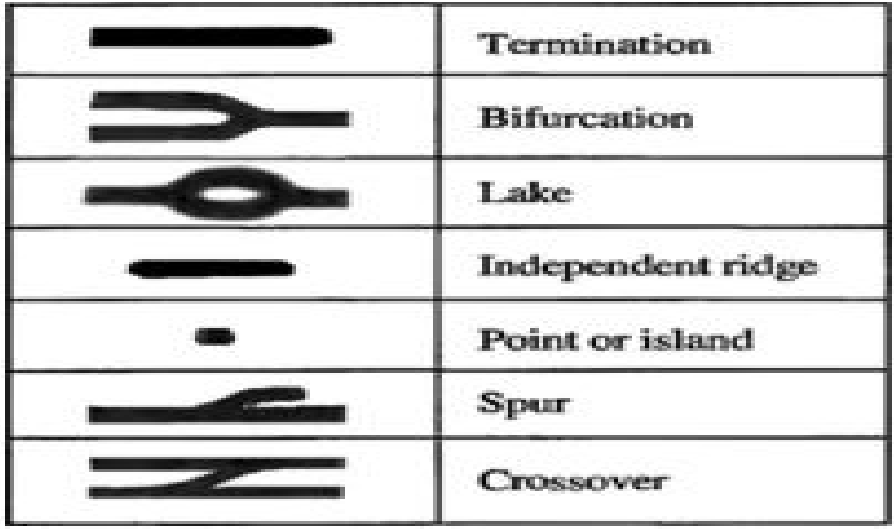

Figure 5. Fingerprint features (Minutiae)
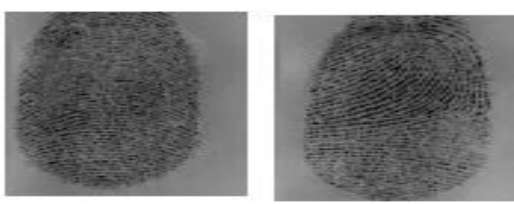

Arc Left loop

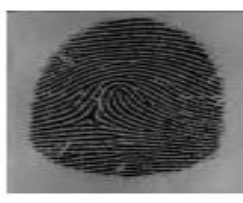

Right loop

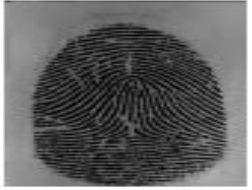

Tented arc

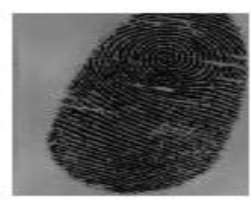

Whorl

Figure 6. Fingerprint classification [68]

The AFRS were developed due to the time consumption associated with the manual methods and overwhelming increase in the fingerprint data. AFRS was proposed to digitize the whole process of fingerprint acquisition and automation of the fingerprint matching techniques incorporating them in an end-to-end system to make the identification and matching process of fingerprint automatic [21, 23]. A typical AFRS consists of five fundamental modules (see Figure 7) including image acquisition, preprocessing (Fingerprint Segmentation, image enhancement) feature extraction, fingerprint matching and classification [20, 21].

The fingerprint image acquisition stage involves the capturing of fingerprint image either in an online mode (using optical fingerprint reader) or offline mode (using ink on the finger and make impression on a white paper and scans the fingerprint) [64]. The pre-processing stage enhances the clarity of the captured fingerprint image ridge structure by removing unwanted data such as noise and reflection [69]. Preprocessing methods includes segmentation, enhancement, orientation extraction, binarization, and thinning [70]. The next stage involves extraction of features from the preprocessed fingerprint image. A fingerprint feature extraction algorithm usually locates, measures, and encodes ridge endings and bifurcations in the fingerprint. There are different algorithms that are used to extract features based on the three levels (global, local, fine details) of fingerprint features, however, 
minutiae(local) feature extraction is commonly used Peralta, 2015 [64, 70]. Minutiae extraction is divided into two, manual extraction based on knowledge based and DL method which extract features automatically from the input fingerprints which is used in AFRS [71].

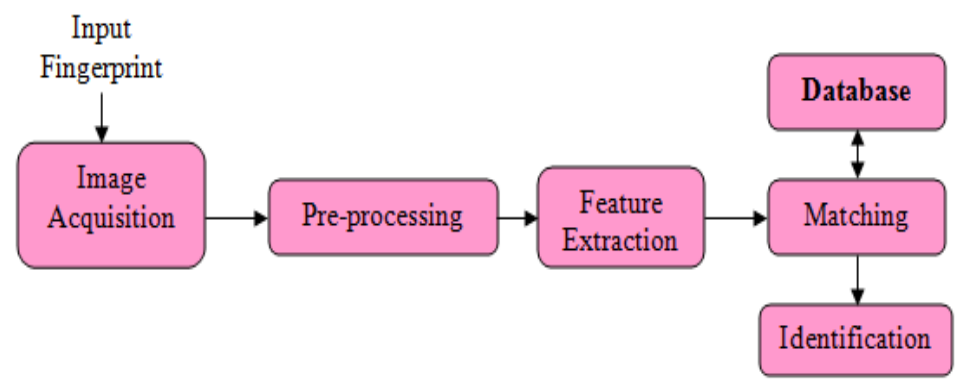

Figure 7. Typical fingerprint recognition steps [69]

The classification or matching stage of AFRS involves comparing the extent of sameness between the pool of enrolled fingerprint features from the database and the extracted features. Based on the degree of match threshold a fingerprint sample is either assigned matched or no matched and real or fake[64]. This paper presents studies that applied DL at various stages of AFRS and at all the levels of fingerprint features to enhance the quality of fingerprint images and to improve recognition performance.

\section{Methodology}

The methodology adopted in this review paper is a systematic literature review (SLR) as proposed in [72]. The SLR is summarized into three major phases as discussed below (also see Figure 8):

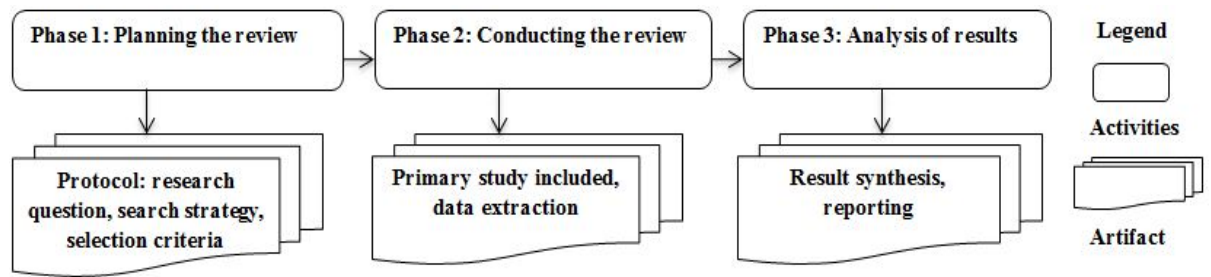

Figure 8. The SLR Process

\subsection{Planning the review}

The planning of the review involves review protocol which explains the procedure in carrying out the actual review. The review protocol comprises of (a) the research questions; (b) the search strategy; (c) study selection criteria; and (d) the data extraction and synthesis of results. 
1) The Research Questions: In respect to the purpose of this study, three research questions were formulated to guide the review.

- $\quad$ RQ1: What are the existing evidences of the application of $D L$ in biometric analysis?

- RQ2: What are the contributions of the application of $D L$ in biometric analysis studies based on DL models, application tasks and dataset?

- RQ3: What are the roles of DL in the training architectures employed in the proposed models?

2) The Search Strategy: The search strategy explains how the best literature are sourced for further analysis. This involves searching electronic databases, performing backward and forward searching to ensure the relevant literature are included for the study [73]. In this review, electronic databases were searched for example, IEEE Xplore (https://ieeexplore.ieee.org/), Science Direct (https://www.sciencedirect.com/) and Springer Links (https://link.springer.com/). The keywords that best describe the title and objective of the study were used in the search string. That is, "deep learning”, "deep neural networks", "CNN" and "fingerprint". However, manual search was employed to include relevant papers that the search string could not fetched. The use of Boolean operators, such as "OR" was used to join synonymous keywords and "AND” to concatenate keywords (see search string below). The searched literature were selectively sampled, this is to ensure only quality papers that satisfy the objective of the study were included. The initial search string was first pilot tested in IEEE Xplore and Science Direct databases to validate that it selects the relevant literature.

Search string: (Deep learning OR deep neural networks OR CNN OR RNN OR LSTM OR DBN OR GAN OR AE) AND (fingerprint)

3) The Study Selection Criteria: The selection criteria include and exclude literature based on their relevance, suitability, and quality for the study. The following are the inclusion criteria, else, they were excluded:

- $\quad$ Primary study that presents evidence of the use of a DL to model fingerprint biometric AND

- $\quad$ Primary study based on empirical evidence AND

- $\quad$ Primary study that is accessible electronically AND

- $\quad$ Primary study that is complete (both methodology and results) AND

- Primary study that is not duplicate (if two versions of a paper are found, the complete version is included) AND

- Primary study that is reported in peer-reviewed journals or conferences or workshops AND

- Primary study between January 1st 2010 and 30th March 2021 inclusive AND 
- $\quad$ Primary study written in English Language.

4) Quality Assessment

Every paper must pass some threshold value of quality in order to be included in the final list of included papers. The quality assessment (QA) was drafted based on the suggestion in [72]. For each of the QA criterion (Table 1), the following scale was used to determine its quality; Yes $(\mathrm{Y})$ represents 1 point, No $(\mathrm{N})$ represent 0 point, and Partial $(\mathrm{P}) \quad$ represents 0.5 point. The expected summation $(\mathrm{x})$ of the scores for each study lies within the interval, $0<=x=>5$ points. The threshold point is calculated by taking the quartile $(5 / 4=1.25)$, by implication, only the studies that score $\boldsymbol{x}>\mathbf{1 . 2 5}$ would be included in the final list. A form was designed to include information to be extracted such as, the publication details, titles, authors, and years. The fields were provided for the application task, DL model, DL model performance, training architecture, and datasets. These data correspond to the research questions and consequently helped in the answering of the research questions as well.

Table 1. QA Criteria Checklist

\begin{tabular}{|c|c|c|}
\hline ID & Quality Assessment Criteria & Rating \\
\hline QA1 & $\begin{array}{l}\text { Was the study objective described in a clear and appropriate } \\
\text { way? }\end{array}$ & $\mathrm{Y}|\mathrm{N}| \mathrm{P}$ \\
\hline QA2 & $\begin{array}{l}\text { Were the methods or techniques used in primary studies } \\
\text { reported clearly? }\end{array}$ & $\mathrm{Y}|\mathrm{N}| \mathrm{P}$ \\
\hline QA3 & $\begin{array}{l}\text { Were the data source, model performance and model } \\
\text { architecture used in the primary study reported clearly? }\end{array}$ & $\mathrm{Y}|\mathrm{N}| \mathrm{P}$ \\
\hline QA4 & Was the study proposal evaluated/validated? & $\mathrm{Y}|\mathrm{N}| \mathrm{P}$ \\
\hline QA5 & Were the results reported clearly? & $\mathrm{Y}|\mathrm{N}| \mathrm{P}$ \\
\hline
\end{tabular}

\subsection{Conducting the review}

This section explains the procedure followed to obtain the included papers. Firstly, the search string was applied on the selected electronic databases. The flowchart in Figure 9 clearly shows the process of literature search, selection, screening, eligibility, and inclusion. The search string was applied on the databases (e.g. IEEE Xplore, Science Direct and Springer links) and $n=68195$ papers were identified. In light of the inclusion criteria stated in section 3.1 sub-section $\mathrm{C}$, the papers were screened. Unrelated papers were removed including review papers, book chapters, posters, letter to the editor and duplicates. The successful screen numbers for the next stage was $n=57$. The papers were checked for eligibility and only $\mathrm{n}=37$ papers passed the stage and were included for data extraction phase. Number of $n=13$ papers were manually searched and were added to the included papers making a total of $n=50$ included papers in the study. Fig. 10a shows the distribution of papers reviewed across the years considered in this review (2010-2021). It can be observed that there is a persistent rise on the number of published papers based on DL to analyze fingerprint biometric images. Based on this 
review no paper was found from the year 2010 to 2012. In 2013, a single (1) paper was found followed by 2014 with three (3) papers published. But in 2015, no paper was found that applied DL to analyze fingerprint images based on this review. Starting from 2016, there was a slight rise of application of DL in analyzing fingerprint images, with five (5) papers published in 2016, nine (9) papers published in 2017 and eleven (11) papers published in 2018. However, there was a drop to seven (7) papers in 2019 but quickly shows rise of interest in the application of DL in fingerprint biometric analysis in 2020 with thirteen (13) papers published. In 2021 (early June), only one paper was included. Fig 10b shows the number of papers published in conferences, journals, and workshops/Symposiums based on this study. It can be observed that 27(54\%) are conference papers, 22(44\%) are journals while $1(2 \%)$ is a workshop paper.

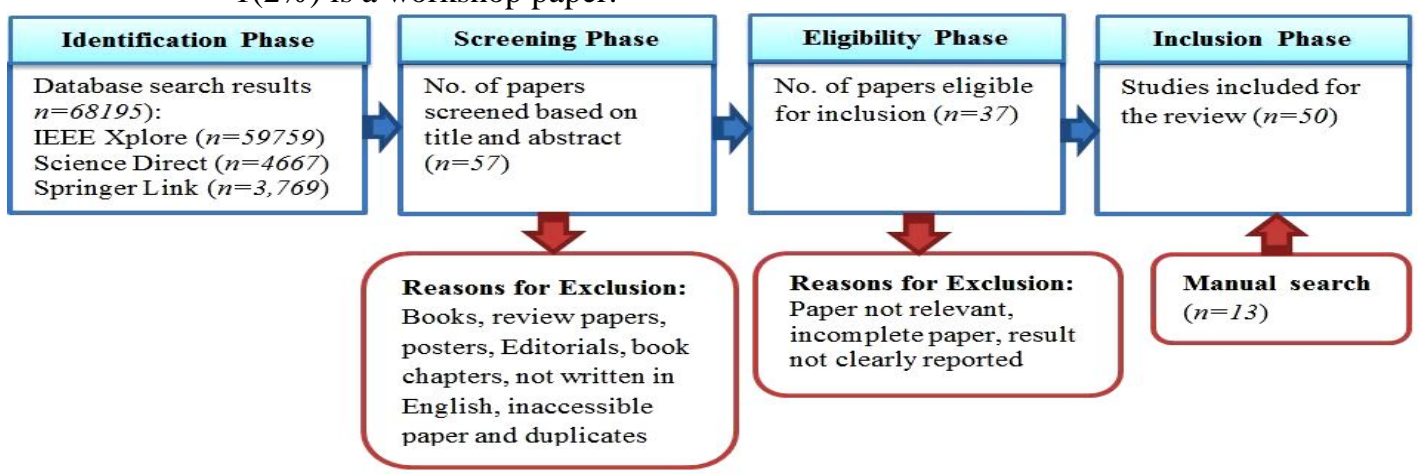

Figure 9. Literature search, screening, eligibility, and inclusion process

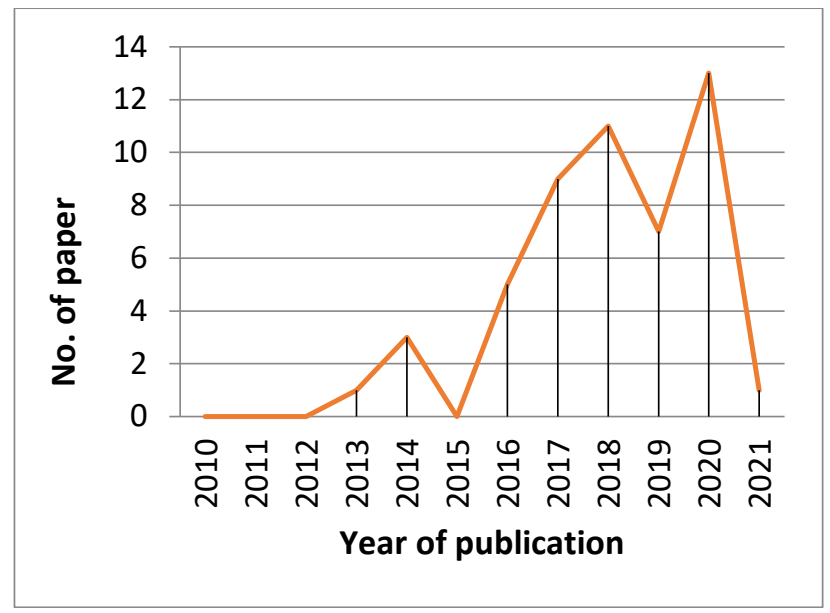

Figure 10(a). No. of papers published across years 


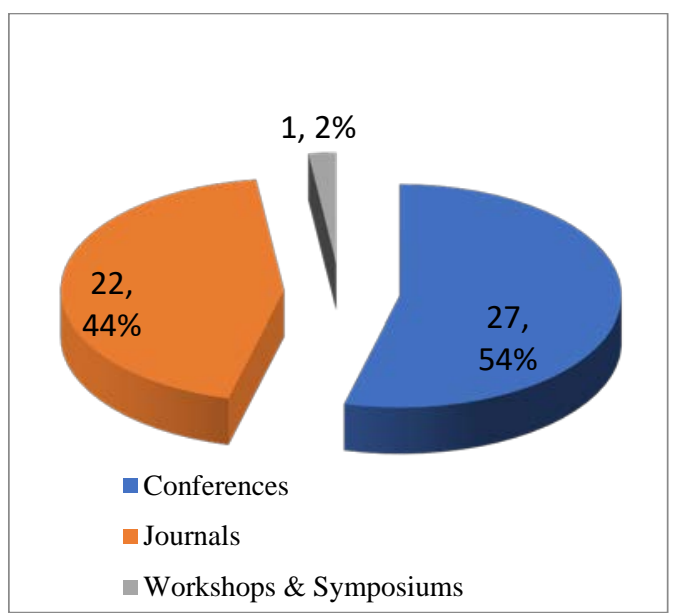

Figure 10(b). No. of conference, journal \& workshop papers

\section{Deep Learning in Fingerprint Biometric Authentication}

\section{RQ1: What are the existing evidences of the applications of $D L$ in biometric analysis?}

Research question 1 seeks to find out the evidence of the application of DL models to carry out various fingerprint biometric analyses. This section presents the primary studies that apply DL in fingerprint biometric analysis. The discussion details the objective of each study, the deep network used to model the fingerprint images, the application task, dataset used to test the model and the performance of the proposed model.

\subsection{Application of Convolutional Neural Network in fingerprint image analysis}

A method based on CNNs was proposed by [74] for liveness detection of fingerprint from fake ones. The authors opined that texture is the most suitable traits to be considered in fake fingerprint detection. The modified CNN method used fire module as used in SqueezeNet and the gram module for texture extraction. However, the authors modified the classical CNN to contain few parameters to enable usage in limited memory devices like smartphones. The CNN model automatically accepts data as input without preprocessing and classifies the fingerprint as fake or real. The Livenes Detection Competition (LivDet) 2011, 2013, 2015 datasets were used to test the model and it achieved an average detection error (ADE) of 2.61\%.

Another liveness detection study was proposed by [75] where the authors used pre-trained CNN based models (CNN-VGG, CNN-Alexnet and CNN with random weights). The models were compared with classical Local Binary Patterns (LBP). The study presented the feasibility of transfer learning and the effectiveness of data augmentation in accurately classifying fingerprints images. Several test were conducted using the LivDet 2009, 2011 and 2013 data with 50,000 real and fake fingerprints images. The result achieved the best average classification error (ACE) of $971 \%$. A method based on CNN (VGG-16) was proposed by [76] for fingerprint and signature authentication systems. The proposed model was tested using the Raspberry Pi 3b and achieved an accuracy of $96.5 \%$ (fingerprint). A study proposed 
by [77] used CNN with random weights and LBP as feature extractors. Then the extracted features after undergoing PCA analysis were fed into the SVM classifier for learning. The CNN model achieved the best performance with $4.71 \%$ ACE when tested using the liveness detection competition (LivDet) in the years of 2009, 2011 and 2013 data.

Fingerprint classification method using CNN was proposed in [78] to classify fingerprint and reduce penetration rate in the database. The modified proposed CNN method was trained on the public National Institute of Standards and Technology (NIST)-DB4 and achieved accuracy of 99\%.

A study conducted by [65] proposed fingerprint livenes detection method to identify fake and live images of fingerprints using CNN architecture. The method applied a modified CNN in an end-to-end approach and as a feature extractor. The extracted features were passed into machine learning classifiers (K-Nearest Neighbors, Logistic Regression and Naïve- Bayes classifier) for the detection. The methods were evaluated on the LivDet 2019 database, and the performances were compared with classical algorithms. The 3-layered CNN produced the best performance with accuracy of $95.3 \%$. However, both the $\mathrm{CNN}$ and the $\mathrm{CNN}+\mathrm{Naïve}$ Bayes method produced the best result in terms of F1-score (0.953).

A study proposed by [79] demonstrated the robustness of CNN based network (VGG16-based deep network) in classifying low quality fingerprint images. Fingerprint images are mostly contaminated with some distortions such as blurriness, dryness, presence of dots, physical damage, and wetness. Transfer learning using VGG16 was employed and was evaluated using a database consisting of 2000 images for training, privately collected for the study. The data were labeled by experts from the GEYCE Biometrics Company. The performance of the CNN based network were 93\% for dry fingerprint classes and $84 \%$ for blurred fingerprint classes. These results show superiority of the DL method to classify low quality fingerprint images against the baselines classifiers (SVM and RF classifiers). However, due to the limited dataset, data augmentation was utilized to reproduce more fingerprint images for evaluation.

A study conducted by [80] proposed fingerprint classification method using two pre-trained CNN based networks (VGGF and VGG-S). Using the approach in transfer learning, the networks were fine-tuned on the NIST-DB4 database. It achieved an accuracy of $94.4 \%$ using VGG-F and $95.05 \%$ when using VGG-S.

A study by [81] proposed a CNN based network (Res-FingerNet) for automatic fingerprint classification. The method is robust in extracting more abstract and global features from fingerprint images. More so, to tackle the problem of intra-class variance and enlarge inter-class variance of the fingerprints, the method applied center loss in the network training stage to ensure the learned deep features are more discriminative and it produced noticeable improvement in classification accuracy (1.5\% increase). The network was evaluated on NIST-DB4 benchmark database and it achieved a classification accuracy of $97.9 \%$. Fingerprint orientation filed is one of the major features of the fingerprint that enhances accurate recognition of fingerprint. Fingerprint orientation represents the trend of the ridge flow of fingerprint. These features of fingerprint help in low quality fingerprint segmentation and enhancement [82]. A study by [26], utilized fingerprint orientation filed to recognize fingerprint images using a CNN-based network (Densenet). The schemes achieved a good average recognition rate of $95.64 \%$.

Latent fingerprint has been an old age piece of evidence used for criminal investigations in forensics and other security agencies like the FBI. However, latent 
fingerprints are not readily usable due to distortions and other interferences. Studies have been conducted to segment areas of interest to detect the criminals. The study in [83] proposed a method based on CNN for latent fingerprint segmentation task. They proposed the method as a classification problem which classifies either fingerprint or background. They carried out the experiments using the popular NIST SD27 latent database that they compared with other methods. The method was found to be better than the existing models on the good, bad and ugly quality images from the NIST SD27 database for visual inspection. The method has a better overall segmentation accuracy of $11.31 \%$.

Enhancement method is used to improve the quality of latent fingerprints before feeding into the AFRS to increase identification rate of the latent fingerprint [84]. A CNN based method was proposed (FingerNet) by [84] for latent fingerprint enhancement. The method analysed the input latent fingerprint pixels-to-pixels in an end-to-end fashion. The evaluation of the proposed method using NIST SD27 datasets yielded the best identification rate with a reduced inference speed of 0.7 better than the existing methods.

An automated latent fingerprint recognition was proposed by [85]. The authors used CNN to capture the latent fingerprint patterns for recognition. Two minutiae templates and one texture template, were utilized which help to extract complementary information from latent fingerprint. The authors tested the model on two public databases NIST SD27 and WVU DB. The results of rank-1 identification accuracies achieved $64.7 \%$ (on NIST SD27) and $75.3 \%$ on WVU DB against a reference database of $100 \mathrm{~K}$ rolled prints.

A study by [86] proposed a DL method based on CNN called FinSNet for overlapped fingerprint separation task. The method is based on representation learning to enhance the retrieval accuracy. The proposed method performed the fingerprint separation with a single inference thereby improved the computational efficiency compared to conventional methods. The Tsinghua overlapped fingerprint datasets (Tsinghua OLF) was used to evaluate the method. The method achieved over $80 \%$ separation rate. A fingerprint classification method was proposed by [15]. This method first preprocessed the input fingerprint images using histogram equalization, Gabor enhancement and fingerprint thinning. The pre-processed fingerprints are input into a deep CNN for classification. The proposed method was evaluated with datasets consisting of 280 samples and accuracy of $98.21 \%$ accuracy was achieved.

A study by[87] proposed a patch-based deep CNN (P-DCNN) method for the detection of double-identity fingerprint enrolled in an electronic database. This paper generated owned dataset and made it public, making the first public double-identity fingerprint dataset. The generated dataset consists of 450 double-identity fingerprints. The proposed method proved to be effective than the compared methods with EER of $2.08 \%$. Studies that segment fingerprint area of interest have been conducted to segment interested regions during the preprocessing stage for matching. A study by [88] proposed a fingerprint ROI segmentation using two CNN based methods, AlexNet and LeNet. The AlexNet produced the best performance when evaluated on the Fingerprint Verification Challenge (FVC) 2002 dataset that added Gaussian noise. The proposed method was tested against another method that used Fourier coefficients without noise, the DL method yielded the best performance with an Err of 0.0392, hc of 0.9402 and mc of 0.0807 .

A study by [89], proposed an explainable fingerprint ROI segmentation. Fingerprint ROI modules are critical in fingerprint segmentation systems. These modules 
capture area of interest of fingerprint and perform analysis to remove unwanted features. However, according to [89], previous methods are mostly uncertain and without confidence. They proposed model based on Unet, a CNN based method called DRUnet (Recurrent Unet with Dropout). They argued that by modeling the uncertainty which will later be used in the training dataset would make the model to possess attention mechanism which would enhance segmentation task. The FVC database was used to evaluate the model against state-of-the-art models on semantic fingerprint segmentation. The proposed method produced the best results using erroneously classified patch percentage (Err) of 0.0173, Hit Coefficient (HC) of 0.9949 and Mistake Coefficient (MC) of 0.0313.

Poor quality images were sometimes acquired during fingerprint image acquisition stage due to factors such as the sensor type, condition of finger, and contact between sensor and finger. Fingerprint enhancement techniques are employed to improve the quality of the fingerprint structure for a more accurate detection or classification tasks [20]. A study conducted by [20], proposed a multi-CNN method called OFFIENet for fingerprint image enhancement task. The proposed method enhanced the corrupted fingerprint images by reconstructing of fingerprint orientation field and fingerprint ridge structure simultaneously. The paper generated corrupted fingerprint image dataset associated with the ground truth to evaluate the model. The performance of the proposed multi-CNN method was found to be better than the compared methods using FVC fingerprint datasets. More so, OFFIENET-tri+VeriFinger produced the best accuracy of $84.10 \%$ against the compared methods.

The fingerprint indexing methods have been used to enhance the performances and speed of the fingerprint identification and matching tasks. Due to the increase in number of fingerprints biometric data and applications, large databases are being set up to keep record of the datasets. However, it has further made the AFRS more challenging than ever. The implications in running time consumption and memory during fingerprint matching introduce another challenge for AFRS [90]. To contend with this challenge, a fingerprint indexing was employed to narrow the search space to similar template. Then, a precise but slow fingerprint matching approach is used to return the truly matched one. A deep compact binary minutia cylinder code (DCBMCC) was proposed by [90] for fingerprint indexing. The method first analyzed minutia cylinder code (MCC) representation. Then, a novel fingerprint indexing method based on DL to learn DCBMCC was proposed. This was to improve the deficiency of MCC high-dimensional, strongly redundant, less discriminative and lossy binary quantization problems. Finally, a MIH-based fingerprint indexing method was proposed which further speeds up the exact search in the Hamming space. The proposed DCBMCC-MIH (s) was tested against DCBMCC-LinScan (s) which proved more efficient and much faster on 64-bit and 128-bit cases.

A study by [91] proposed fingerprint indexing method on representation learning to enhance retrieval accuracy and efficiency in the ever increasing fingerprint databases. First, the method applied deep CNN (DCNN) to learn minutiae discriminator for representing local ridge structures. Subsequently, a method to aggregate various number of minutiae-centered deep convolutional (MDC) features to form a fixed -length, discriminative and compact feature vector for indexing from one fingerprint. The proposed method was evaluated using five databases from NIST and FVC2000 databases and the results produce the best performance in terms of accuracy and efficiency. A partial fingerprint matching algorithm was proposed by 
[92] based on phase-only correlation (POC)-based method and deep CNN. The method first aligned two fingerprints and the features are extracted with the algorithm based on POC. The deep CNN was employed with spatial pyramid pooling to checkmate if the extracted regions come from the same finger or not. The method was evaluated and compared with the existing methods on two databases, FingerPass DB7 dataset and in-house database. The performance based on EER was $4.01 \%$ and $1.32 \%$ respectively.

Another partial fingerprint algorithm was proposed by [93] using a DL method based on ResNet. Cross entropy function and contrast-Loss function are the two loss functions used to design the residual network. The method employs the feature vectors of fingerprint and classification information to achieve a good classification performance. The proposed method was evaluated using NIST-DB4 and self-built dataset NCUTFR (North China University of Technology-Finger Recognition). The performance was evaluated using NIST-DB4 that was partition into two, NIST-DB4_F (contains the first impression of each fingerprint image) and second impression (NIST-DB4_S) and obtained classification accuracy of $91.80 \%$ (on NIST-DB4_F) and 93\% (on NIST-DB4_S).

The detection of intruders and criminals in country borders is central for preventing international crimes. Sometimes a criminal can alter his fingerprint in an attempt to by-pass security systems. A study by [94] conducted a research towards detecting altered fingerprints. The paper presented a novel database called Coventry Fingerprints Dataset (CovFingDataset) and made it public. A total of 55,249 images with three levels of alteration for z-cut, obliteration and central rotation synthetic alterations were provided. The proposed method identifies and detects the real fingerprint from the altered fingerprint and the type of the fingerprint. The proposed method although performed a bit less than pre-trained ResNet with $98.55 \%$ against $99.88 \%$ accuracy, the method achieved $100 \%$ precision and recall score on real images, making it better for real-time applications.

A study by [6], proposed a CNN architecture for the detection of spoofed fingerprints for authentication tasks in financial applications and banking. The input fingerprints images to the proposed method were preprocessed using contrast enhancement using histogram equalization. The proposed method was evaluated using the FVC2006, ATVSFFp DB, Spoofing-Attack Finger Vein Database, LivDet 2013, LivDet 2015 datasets. It achieved a high-performance detection rate, with more than 99\% accuracy. A study by [95] proposed a fusion of network of deep features extracted using deep CNN and handcrafted features for concealable biometrics. An integrated biometric recognition was used including face, iris, palm print, fingerprint, and ear biometrics. Various handcrafted methods were employed such as histogram of oriented gradients (HOG), oriented rotated brief (ORB), local binary patterns (LBPs), scale-invariant feature transform (SIFT), and speed-up robust features (SURF). Furthermore, dimensional reduction methods such as independent component analysis (ICA) or principal component analysis (PCA) were employed on the handcrafted features for consistency with the deep features. The proposed method is evaluated on the CASIA Fingerprint dataset produced the best results using SIFT and ICA for fingerprint recognition with accuracy of 96.69, specificity f 96.77, precision of 94.88, recall 95.94 and F1-score of 95.4.

A study to improve the quality of fingerprint digitally was proposed by [96]. The paper proposed a deep network to avoid the time consumption during physical recapturing of fingerprint. The proposed method used Cycle-Consistent Adversarial Networks (Cycle-GAN) for an unpaired image-to-image translation that was used 
for translating images from distorted domain to undistorted domain. The authors proposed a CNN based deep network (VGG16) to evaluate the correct score of undistorted fingerprint using a real life datasets from GEYCE biometrics. The method achieved $94 \%$ quality improvement for wetness fingerprint.

A fingerprint pattern identification and classification was proposed by [67]. The study proposed a six category fingerprint classes unlike the conventional four-category fingerprint classes. A CNN based network (FCTP-Net) was proposed to evaluate the robustness of the proposed method. The method was evaluated using NIST datasets and the six-category fingerprint database produced accuracy of $94.87 \%$ and $92.9 \%$ using the four-category fingerprint database. The paper demonstrated the suitability and stability of FCTP-Net in identifying six-category fingerprint database precisely.

Fingerprint classification study by [97] proposed a method to classify fingerprint image into four categories of the left-right hand, sweat-pore, scratch and finger type category using DL approach. Five DL models based on CNN (classic CNN, Alexnet, VGG-16, Yolo-v2 and Resnet-50) were used. The study used dataset from Cambodia to train and validate the models. Subsequently, the Korean fingerprint images were used to test the models. The overall performance accuracy was achieved by yolo-v2, $90.98 \%$ for left-right hand, $78.68 \%$ for scratch, $66.55 \%$ for fingers and $91.29 \%$ for sweat-pore (Resnet-50). Transfer learning was used to train network on the source dataset on a new dataset with the same or different characteristics. A study by [25] proposed a pre-trained CNN (ResNet50) for fingerprint recognition task. They fine-tuned RestNet50 network was evaluated using polyU fingerprint database and obtained a better accuracy rate of $95.7 \%$ when compared with the previous methods on the same database.

Fingerprint pores have been found helpful to improving the performance of the AFRS. This is because of the recent development of high resolution fingerprint images that becomes common. A method to recognize and extract pores in a small image block was proposed by [98]. Experiments were carried out using PolyU HRF DBI and their method produced better result than the existing methods with $88.6 \%$ pore detection rate. The proposed method was also tested for the fingerprint matching and produced a low EER of 3.66\%. A new deep feature descriptor for pore representation in direct pore matching was proposed by [99] called DeepPoreID. The proposed method was evaluated using two databases, DBI and DBII. The proposed method outperformed existing methods in EER, FMR1000 and time consumption. In another study, [70] proposed a method to match aligned fingerprint images. Based on the characteristics of the fingerprint images, a CNN network based on VGG-16 called Finger ConvNet was proposed. The proposed method was evaluated on two lab databases and two public datasets, NIST sd04 and NIST sd14. It was tested using FVC public datasets as benchmark database. Although, the proposed network produces less in some database, it outperformed in some databases and achieved overall performance in terms of speed (most 5 times better than the fastest compared methods). The low performance in the proposed method was attributed to the fact than it does not use artificial features as in the conventional methods; as such the proposed network is robust regardless of the dataset. A study presented in [100] considered two deep networks based on CNNs which they used for automatic classification of fingerprint. The proposed models are CaffeNet, which is a variant of the popular AlexNet and network with a smaller units than CaffeNet which was aimed at simplifying the search space of the neural network training to accelerate the convergence. The DL models were tested using NIST-DB4, SFinGe databases, and 
obtained a classification accuracy of 98.94\% CaffeNet and 99.07\% (proposed network). Both the proposed networks outperformed existing methods on accuracy and penetration rate. Run time was also improved as a result of combined feature extraction and classification in an end-to-end training architecture.

A study by [101] proposed a method for fingerprint pattern classification which used VGGNet, a CNN based network. Three modifications of VGGNet were combined with ensemble model to improve accuracy. Also, batch normalization was applied to curtail the limitations of ensemble model such as overfitting and a slow learning speed by applying it between the convolution operation and the activation function operation. The proposed networks were tested on the FVC 2000, 2002, 2004 databases and the best model produced $98.3 \%$ accuracy level.

Minutiae are essential features during fingerprint recognition process. However, traditional methods are tedious and time consuming because they involved hand-defined preprocesses such as binarization, thinning, and enhancement [102]. Deep networks can extract minutiae features from the raw fingerprint images automatically without any pre-processing stage. The proposed model by [102] set up a study for the automatic extraction of minutiae from the fingerprints. A novel minutiae extraction approach based on multiple deep CNNs (the JudgeNet and the LocateNet) was proposed. The proposed method compared with existing methods produced the best total error (dropped minutiae + false minutiae) of $12.91 \%$ using the sampled images from the Chinese criminal investigation fingerprint database. The proposed method was also compared with a well-known commercial system, GAFRS, and produced over all best performance with 94.59, 91.63 and 93.09 of precision, recall and $\mathrm{F} 1$ score respectively.

A study conducted by [71] proposed a method for fingerprint feature extraction task. They combined domain knowledge and deep networks (FingerNet) for a robust generalization. The proposed method efficiently and reliably extracted minutiae on both rolled/slap and latent fingerprints. Two databases NIST SD27 and FVC 2004 database were employed to evaluate the generalization ability of the proposed method. The proposed method produced better performance in terms of extraction and identification.

In a study presented by [103] a CNN based deep network called MENet for Minutiae Extraction is proposed. The proposed deep network was compared to the ground truth minutiae and achieved success rate above $80 \%$. The network was trained on the 2000, 2002, and 2004 FVC datasets to obtain minutiae miss-rate of only $14.2 \%$.

In recent years, contactless $3 \mathrm{D}$ fingerprint recognition is gaining acceptance because of its hygienic, accurate and ubiquitous nature. A study conducted by [104] demonstrated the effectiveness of deep network in contactless to contact based fingerprint images matching task over the shallow techniques. In order to deviate from the problem of loss of fingerprint features in the conventional convolution and pooling operations of $\mathrm{CNN}$, the authors proposed to incorporate specific features (minutiae of fingerprint) and regions of the fingerprint ridge map based on fingerprint core point with ridge images as input to their proposed multi Siamese networks. Their proposed network was experimented using PolyU Contactless to Contact-based fingerprint database and obtained better performance on equal error rate (EER) of $8.39 \%$ which is better than the existing methods.

Partial 3D fingerprint is a common problem using the contactless 3D fingerprint technology which often degrades the fingerprint matching performance accuracy. In a study conducted by [105], a contactless and partial 3D fingerprint recognition method was proposed. A CNN based model consisting of one fully convolutional 
network (based by fine-tuning VGGNet) for fingerprint segmentation and three Siamese networks to learn multi-view 3D fingerprint feature representation. Two databases, contactless 3D fingerprint database and multi-view contactless fingerprint database were used to evaluate the proposed multi-view CNN network. The EER of the proposed network (7.27\%) outperformed minutiae based $(17.33 \%)$ and fine-tuning Resnet (9.58\%). Table 2 shows the summary of fingerprint biometric schemes based on CNNs.

Table 2. Summary of DL application in fingerprint Biometric based on CNN

\begin{tabular}{|c|c|c|c|}
\hline Reference & Application task & Dataset & Performance \\
\hline $\begin{array}{l}\text { Park, Cui, Kim } \\
\text { and Kim [74] }\end{array}$ & $\begin{array}{l}\text { Fingerprint } \\
\text { liveness detection }\end{array}$ & $\begin{array}{l}\text { LivDet 2011, } \\
\text { 2013, } 2015 \text { data }\end{array}$ & $\mathrm{ADE}=2.61 \%$ \\
\hline $\begin{array}{l}\text { Nogueira, de } \\
\text { Alencar Lotufo } \\
\text { and Machado [75] }\end{array}$ & $\begin{array}{l}\text { Fingerprint } \\
\text { liveness detection }\end{array}$ & $\begin{array}{l}\text { LivDet 2009, } \\
2011 \text { and 2013 } \\
\text { data with 50,000 } \\
\text { real and fake } \\
\text { fingerprints } \\
\text { images }\end{array}$ & $\mathrm{ACE}=97.1 \%$ \\
\hline $\begin{array}{l}\text { Nogueira, de } \\
\text { Alencar Lotufo } \\
\text { and Machado [77] }\end{array}$ & $\begin{array}{l}\text { Fingerprint } \\
\text { liveness detection }\end{array}$ & $\begin{array}{l}\text { Livdet 2009, } \\
2011 \text { and } 2013 \\
\text { data }\end{array}$ & $\begin{array}{l}\text { Accuracy }=95.2 \% \\
\text { ACE }=4.71 \%\end{array}$ \\
\hline $\begin{array}{l}\text { TK, } \\
\text { Vinayakumar, } \\
\text { Sowmya and } \\
\text { Soman [65] }\end{array}$ & $\begin{array}{l}\text { Fingerprint } \\
\text { liveness detection }\end{array}$ & $\begin{array}{l}\text { LivDet } 2019 \\
\text { Database }\end{array}$ & $\begin{array}{l}\text { Accuracy= 95\% } \\
\text { (Test) }\end{array}$ \\
\hline $\begin{array}{l}\text { Goel, Puhan and } \\
\text { Mandal [87] }\end{array}$ & $\begin{array}{l}\text { Double-Identity } \\
\text { Fingerprint } \\
\text { Detection }\end{array}$ & $\begin{array}{l}\text { Database of } 450 \\
\text { double-identity } \\
\text { fingerprints }\end{array}$ & $\begin{array}{l}\mathrm{EER}=2.08 \\
\text { FMR100=2.24\%, } \\
\text { zero FMR=3.2\%, } \\
\text { and zero } \\
\text { FNMR }=31 \%\end{array}$ \\
\hline
\end{tabular}

Shehu,

Ruiz-Garcia, Fingerprint

Palade and James alteration detection

CovFingDataset Accuracy $=98.55 \%$

[94]

Arora and Bhatia

Fingerprint

[6]

Spoofing Detection

FVC2006,

ATVSFFpDB,

Spoofing-Attack

Finger Vein $\quad$ Acc $>99 \%$

Database,

LivDet 2013,

LivDet 2015

Fingerprint

Shuping and Feng Fingerprint

orientation field

data and

fingerprint

images

Minaee, Azimi

Fingerprint

PolyU

Average recognition rate $=$ $85.64 \%$.

Accurate rate $=$ 
and Abdolrashidi recognition

[25]

Abdellatef,

Omran, Soliman,

Ismail, Abd

Elrahman, Ismail,

Rihan, Abd

El-Samie and Eisa

[95]

Cao and Jain [85]

Lin and Kumar

[105]

Amruth,

Gopinatha,

Gowtham, Kiran

and Harshalatha

[76]

Yoo, Cho, Lee,

Chae, Lee and

Lee [86]

Ge, Bai, Liu, Liu

and Zhao [81]

Fingerprint

classification

Michelsanti, Ene,

Guichi, Stef,

Nasrollahi and

Moeslund [80]

Pandya, Cosma,

Alani,

Taherkhani,

Bharadi and

McGinnity [15]

Tertychnyi,

Cancelable

recognition

systems

recognition

fingerprint

recognition

Fingerprint

Overlapped

fingerprint

separation

\section{Fingerprint}

classification

Fingerprint

Classification

Low quality
Latent fingerprint

Contactless and

partial 3D

authentication

\section{NIST SD4 \\ database}

Contactless 3D

fingerprint

Database and

Multi-view

Contactless

Fingerprint

Database

Image dataset

with labeled

signature and

fingerprint

images

Tsinghua OLF

and the Tsinghua

simulated

overlapped

fingerprint

dataset

NIST-DB4

database

Accuracy $=96.69$, Specificity $=$

96.77, precision

$=94.88$,

Recall=95.94 and

F1-score $=95.4$.

Rank-1

identification

accuracies=

64.7\% (NIST

SD27) $75.3 \%$ for

the WVU DB

$\mathrm{EER}=7.27 \%$

Accuracy $=96.5 \%$

(fingerprint)

90.21\% (signature)

\section{Over $80 \%$}

separation rate

Accuracy $=97.9 \%$

Accuracy $=94.4 \%$

(VGG-F) and

95.05\% (VGG-S)

Error rates $=5.6 \%$

(VGG-F) and

4.95\% (VGG-S)

Dataset created

for 560 samples

from 56 users

Accuracy= 98.21\%

Database

Accuracy $=93 \%$ 


$\begin{array}{llll}\begin{array}{l}\text { Ozcinar and } \\ \text { Anbarjafari [79] }\end{array} & \begin{array}{l}\text { fingerprint } \\ \text { classification }\end{array} & \begin{array}{l}\text { consisting of } \\ \text { 2000 sample } \\ \text { data labeled by } \\ \text { experts from }\end{array} & \begin{array}{l}\text { (dry fingerprint } \\ \text { classes) and 84\% } \\ \text { (blurred fingerprint } \\ \text { classes }\end{array} \\ & \begin{array}{l}\text { GEYCE } \\ \text { Biometrics }\end{array} & \\ & & & \text { Accuracy=92.9\% } \\ & & & \begin{array}{l}\text { (4-category } \\ \text { fingerprint }\end{array} \\ & & \text { Fatabase) }\end{array}$


Karabulut,

Tertychnyi,

Arslan, Ozcinar,

Nasrollahi, Valls,

Vilaseca,

Moeslund and

Anbarjafari [96]

Song, Tang and

Feng [91]

Fingerprint

indexing

Bai, Wang, Zhao,

Wang and Li [90]

Liu, Zhao, Liu

and Shen [99]

Liu, Zhou, Han,

Guo and Qin [70]

Qin, Tang, Han

and Guo [92]

Zeng, Hu and

Xiao [93]

Lin and Kumar
[104]

[104]

Tang, Gao, Feng
Fingerprint

enhancement
Aligned

fingerprints

matching matching matching

Contactless to

Contact-based matching

Fingerprint

Fingerprint
indexing
Fingerprint pore
matching

Partial fingerprint

Partial fingerprint

Fingerprint Images

datasets (testing)

Real life dataset from GEYCE

Biometrics

94\% quality

improvement

NIST: DB4 nat.

NIST144, NIST $\quad 0.26 \%$, DB4

$0.28 \%$ and

4 natural, NIST,

FVC2000 DB2a,

DB3a

DB14, 0.06\%

FVC2000: DB2a

$1.02 \%$, DB3a

$1.21 \%$

5.89 (128-bit)

NIST DB14

0.76 (64-bit)

FVC2000,

$\mathrm{EER}=1.42(\mathrm{DB} 1)$

FVC2002, and 0.51(DBII)

FVC2004

datasets

ID Card

fingerprint

dataset and the

Ten-Finger

Fingerprint Card

fingerprint

dataset

(experiment)

FVC2000,

FVC2002, and

FVC2004

FMR1000=7.69

(DBI) 4.05(DBII)

Proposed method

produced better

performance in

some databases

with overall

matching speed of about 5 times the fastest compared

method

datasets (testing)

FingerPass DB7

dataset and

$\mathrm{EER}=4.01 \%$ and

in-house

$1.32 \%$

database

Accuracy $=91.80 \%$

NIST-DB4 and

(NIST-DB4_F)

NCUTFR dataset and 93\%

(NIST-DB4_S)

PolyU

Contactless to

Contact-based $\quad$ EER $=8.39 \%$

Fingerprint

Database

NIST SD27 and

Better performance 


\begin{tabular}{|c|c|c|c|}
\hline and Liu [71] & minutiae extraction & $\begin{array}{l}\text { FVC } 2004 \\
\text { database set A }\end{array}$ & $\begin{array}{l}\text { was achieved in } \\
\text { terms of precision } \\
\text { and identification } \\
\text { rate }\end{array}$ \\
\hline & Fingerprint & 2000,2002 , and & $\begin{array}{l}\text { Minutiae miss-rate } \\
\text { of only } 14.2 \%\end{array}$ \\
\hline $\begin{array}{l}\text { Darlow and } \\
\text { Rosman [103] }\end{array}$ & $\begin{array}{l}\text { Minutiae } \\
\text { Extraction }\end{array}$ & $\begin{array}{l}2004 \\
\text { FVC datasets }\end{array}$ & $\begin{array}{l}\text { of only } 14.2 \% \\
\text { success rate above } \\
\text { of } 80 \%\end{array}$ \\
\hline $\begin{array}{l}\text { Su, Chen, Wong } \\
\text { and Lai [98] }\end{array}$ & Pore Extraction & PolyU HRF DBI & $\begin{array}{l}\text { EER of } \\
3.66 \% \\
\text { pore detection rate } \\
\text { of } 88.6 \% \text {. }\end{array}$ \\
\hline $\begin{array}{l}\text { Jiang, Zhao, Bai, } \\
\text { Yong and Wu } \\
{[102]}\end{array}$ & $\begin{array}{l}\text { Minutiae } \\
\text { Extraction }\end{array}$ & $\begin{array}{l}\text { Chinese criminal } \\
\text { investigation } \\
\text { fingerprint } \\
\text { database }\end{array}$ & $\begin{array}{l}\text { Total error } \\
=12.91 \%\end{array}$ \\
\hline
\end{tabular}

\subsection{Deep Belief Network for Fingerprint Authentication}

The RBM is used for the development of AFRS for example, a study by [106] proposed a method for fingerprint liveness detection using DBN in an automatic leaning. The model used data augmentation which enhances the accuracy of the live detection model. The proposed DBN models achieved better performance with an ACE of $97.10 \%$. A study proposed by [107] used two continuous RBM (CRBMs) to learn local orientation field patterns and to correct distorted field patterns in noisy fingerprint images. The study used Gabor based algorithms on the learnt orientation field for fingerprint enhancement. The automatic deep network was evaluated based on the number of spurious and missing minutiae generated by the algorithm on the FVC2002 Db3a database and produced spurious and missing minutiae of 26259(62.87\%) and 3592(18.87\%), respectively. the proposed method revealed that it is $9 \%$ less than the hand-marked generated minutiae.

In another study, [108] proposed a convolutional DBN (CDBN) that performs reconstructions of fingerprint images using what the network learns. These reconstructions serve as enhancements of these images. The proposed method was tested on three datasets: FVC 2000 Db1 a, FVC 2002 Db1a and NIST-4 special database and obtained EER of 8.79. The performance of the proposed method outperformed the compared methods. A study to mitigate spoofing attacks on biometric systems was proposed in [109]. The paper used a model based on RBM (Deep RBM + DBM) to differentiate between real and fake fingerprint thereby tackling the problem of spoofing in the system. The method examines the image consistency based on ROI and scaled. The method applied DBM on the fingerprint images to extract deep features while the KNN was used for binary classification. The robustness of the method was evaluated on two livDet databases (LivDet 2013, LivDet 2015) and achieved ACE of 3.6\%. However, the method still struggles to recognize fake images from unknown material and it was evaluated to have the problem of time complexity.

Table 3 shows the summary of the application of RBN DL-based models in fingerprint biometric analyses 
Table 3. Summary of application of DL in fingerprint biometric analyses based on RBNs

\begin{tabular}{|c|c|c|c|}
\hline Reference & Application task & Dataset & Performance \\
\hline $\begin{array}{r}\text { Kim, Park, } \\
\text { Song and Yang } \\
{[106]}\end{array}$ & $\begin{array}{r}\text { Fingerprint } \\
\text { liveness } \\
\text { detection }\end{array}$ & LivDet2013 & $\mathrm{ACE}=97.10$ \\
\hline $\begin{array}{r}\text { Uliyan, } \\
\text { Sadeghi and } \\
\text { Jalab [109] }\end{array}$ & Anti-spoofing & $\begin{array}{r}\text { LivDet 2013, } \\
\text { LivDet } 2015\end{array}$ & ACE of $3.6 \%$ \\
\hline $\begin{array}{r}\text { Sahasrabudhe } \\
\text { and } \\
\text { Namboodiri } \\
\text { [108] }\end{array}$ & $\begin{array}{r}\text { Fingerprint } \\
\text { enhancement }\end{array}$ & $\begin{array}{r}\text { FVC } 2000 \text { Db1 a } \\
\text { FVC } 2002 \text { Db1 a } \\
\text { and NIST-4 } \\
\text { special database }\end{array}$ & $\mathrm{EER}=8.79$ \\
\hline Sahasrabudhe & Learning & FVC2002 Db3a & Spurious= \\
\hline and & Fingerprint & database & 26259(62.87\%) and \\
\hline Namboodiri & Orientation & & missing \\
\hline \multirow[t]{2}{*}{ [107] } & Fields/ & & $=3592(18.87 \%)$ \\
\hline & $\begin{array}{l}\text { Fingerprint } \\
\text { enhancement }\end{array}$ & & \\
\hline
\end{tabular}

\subsection{Autoencoder Applications in Fingerprint Authentication}

One of the most challenges facing most fingerprint recognition systems is the spoofing attack. This attack tries to circumvent a recognition system using artificial replicas of human characteristics similar to the legitimate enrolled trait. Methods based on $\mathrm{AE}$ have been proposed over the years to minimize this attack on fingerprint recognition systems. A study proposed by [110] conducted a fingerprint liveness detection study based on stacked AE with supervised learning. The stacked $\mathrm{AE}$ was analysed and a new RFLD algorithm based on semi-supervised learning stacked AE was proposed. The proposed model was evaluated on two public datasets (LivDet 2011 and 2013), it produce good performance with ACE of 19.62\% (LivDet2013) and 18\% (LivDet2011). Another study based on AE in [111] proposed a deep network for fingerprint classification problem. A sparse AE with three hidden layers was used to model four classes of the fingerprint. The NIST-DB4 database was employed to evaluate the proposed method which achieved accuracy of 93.1\%. In another study, [112] proposed a fingerprint classification method using the stacked sparse AE (SAE) with three hidden layers. The proposed method was able to classify four classes of fingerprint (arch, left loop, right loop, and whorl) with 91.4\% accuracy on the NIST-DB4 database. The performance of the method has demonstrated to produce better performance using a Fuzzy classification method. Table 4 presents the summary of the applications of DL based on AE in fingerprint biometric analyses.

Table 4. Summary of AE in fingerprint biometric analyses

\begin{tabular}{llll}
\hline Reference & $\begin{array}{l}\text { Application } \\
\text { task }\end{array}$ & Dataset & Performance \\
\hline Yuan, Chen, Yu, & Fingerprint & LivDet & ACE=19.62 (LivDet2013) \\
Meng, Cheng, liveness & 2011 and & 18\% (LivDet2011) \\
$\mathrm{Wu}$ and Sun detection & 2013 & \\
{$[110]$} & & &
\end{tabular}




\begin{tabular}{llll}
$\begin{array}{l}\text { Wang, Han, Wu } \\
\text { and Guo [111] }\end{array}$ & $\begin{array}{l}\text { Fingerprint } \\
\text { classification }\end{array}$ & $\begin{array}{l}\text { NIST-DB4 } \\
\text { database }\end{array}$ & $\begin{array}{l}\text { Accuracy }=91.4 \% \\
\text { Accuracy }=98.8 \% \\
\text { method) }\end{array}$ \\
$\begin{array}{l}\text { Wang, Han and fuzzy } \\
\text { Guo [112] }\end{array}$ & $\begin{array}{l}\text { Fingerprint } \\
\text { classification }\end{array}$ & $\begin{array}{l}\text { NIST-DB4 } \\
\text { Database }\end{array}$ & \begin{tabular}{l} 
Accuracy=91.4\% \\
\hline
\end{tabular} \\
\hline
\end{tabular}

\subsection{Generative Adversarial Network Applications in Fingerprint}

The GAN has been used extensively to generate fingerprint images [113, 114]. Approaches based on GAN for fingerprint authentication schemes have generated near real images compared with the original or real data. A study by [113] proposed a method called DC-GAN to generate fingerprint images with similar characteristics and quality to the real fingerprint images. The method achieved a good performance in terms of Frechet Inception distance (FID), with 70.5 score. Two datasets FVC-2006 fingerprint dataset and PolyU fingerprint dataset were utilized to test the Finger-GAN method. Another study by [114] proposed a two folded research for fingerprint generation and presentation attack detection methods. GAN was used to generate synthetically fingerprint images using two steps, namely, master minutia set generation and fingerprint impression creation. The paper also proposed a method based on CNN (squeeze net) for presentation attack. The performance was found to be better than the previous works with ACE of 1.57.

A study to mitigate attacks on fingerprint biometric systems was proposed by [115]. The study proposed a method to demonstrate the vulnerability of skeleton images to presentation attack. The authors applied a technique to extract a skeleton image of fingerprint from the leaked template and then tried to recover the fingerprint that looks as much as the original fingerprint such that it can deceive the fake detection system. The proposed model Pix2Pix model was used in the study. After recovering the fingerprints, the proposed method achieved over $90 \%$ of presentation attack success rate at $0.1 \%$ FMR. Table 5 presents the summary of the application of DL based on GAN in fingerprint biometric analyses.

Table 5. Summary of GAN applications in fingerprint biometric analyses

\begin{tabular}{|c|c|c|c|}
\hline Reference & Application task & Dataset & Performance \\
\hline $\begin{array}{l}\text { Minaee and } \\
\text { Abdolrashidi } \\
\text { [113] }\end{array}$ & $\begin{array}{l}\text { Generate fingerprint } \\
\text { Images }\end{array}$ & $\begin{array}{l}\text { FVC-2006 } \\
\text { fingerprint } \\
\text { dataset, and } \\
\text { PolyU fingerprint } \\
\text { dataset }\end{array}$ & FID $=70.5$ \\
\hline $\begin{array}{l}\text { Kim, Cui, } \\
\text { Kim and } \\
\text { Nguyen } \\
\text { [114] }\end{array}$ & $\begin{array}{l}\text { Fingerprint generation } \\
\text { and presentation attack } \\
\text { detection }\end{array}$ & $\begin{array}{l}\text { LivDet 2011, } \\
\text { 2013, and } 2015\end{array}$ & $\mathrm{ADE}$ rate $=1.57 \%$ \\
\hline $\begin{array}{lr}\text { Lee, } & \text { Jang, } \\
\text { Kim, } & \text { Hahn } \\
\text { and } & \text { Kim } \\
{[115]} & \\
\end{array}$ & $\begin{array}{l}\text { Fingerprint } \\
\text { Scheme }\end{array}$ & $\begin{array}{l}\text { NIST Special } \\
\text { Database } 4\end{array}$ & $\begin{array}{l}\text { over } \quad 90 \% \text { of } \\
\text { presentation attack } \\
\text { success rate }\end{array}$ \\
\hline
\end{tabular}




\subsection{Hybrid Deep Learning Approach for Fingerprint}

An approach whereby proposed biometric system was modeled using a combination of two or more DL models were called ensemble DL approach. A study presented by [116] proposed a method for localization and detection of altered fingerprint in order to avoid obfuscation presentation attack. The first part of their model utilized CNN models (Inception-v3 and Mobilenet-v1) to detect and localize regions of fingerprint alterations. Then, GAN was applied to synthesize altered fingerprints. The authors used 4,815 of altered and 4,815 valid fingerprints from 270 subjects. The result of the experiment showed that the True Detection Rate (TDR) of $99.24 \%$ and a False Detection Rate (FDR) of $1 \%$ can be achieved. However, the performance of GAN model can be improved by fined-tuning it. Table 6 presents the summary of the studies that applied GAN for AFRS.

Table 6. Summary of hybrid approach in fingerprint biometric analyses

\begin{tabular}{llll}
\hline Model/Author & Application area & Dataset & Performance \\
& & & \\
\hline Tabassi, & Fingerprint localization & 4,815 altered and & TDR $=99.24 \%$ at \\
Chugh, Deb & and detection & 4,815 valid & a FDR $=1 \%$, \\
and Jain [116] & & fingerprints from & \\
& & 270 subjects & \\
\hline
\end{tabular}

\section{Discussion and Analysis}

This section presents the discussions on the proposed DL models, the application tasks performed by the model, the benchmark dataset used to evaluate the models' performances and the training architecture employed during model training.

RQ2: What are the contributions of the application of $D L$ in biometric analysis studies based on DL models, application tasks and dataset?

Research question 2 seeks to identify the DL models, the application tasks, and the datasets used in the existing studies of the application of DL in fingerprint analysis.

\subsection{Deep Learning models}

Based on this review, various DL models have been used to analyse fingerprint images to either enhance the quality of the fingerprint images or to improve the performance of the AFRS. Reference to Table 2 and Figure 11, it is obvious that CNN based models received the highest number of applications for fingerprint image analyses. The attraction in the use of CNN based models may be as a result of the robustness of CNN to gray variation, noises and a certain degree of deformation [20] [70]. More so, CNNs models are suitable for image classification and because fingerprint produce image data [52]. In addition, this may be attributed to the success and popularity of CNN-based variants for image classification such as ResNet, LeNet, AlexNet, VGG-Net, SqueezeNet, googLeNet etc. These features of CNNs explain the wide application of CNN-based models in biometric recognition systems such as face recognition, iris recognition and fingerprint recognition [3, 27]. Other DL identified in this study to model fingerprint images are DBN, AE and GAN. However, based on this study, no single paper among the included papers was found to be based on deep recurrent neural networks (RNN) [117] for fingerprint biometric analyses. This may be attributed to the inherent characteristics of RNN 
based models which are well-known for processing sequential data like speech and time-series data. Therefore, not robust for image processing [118].

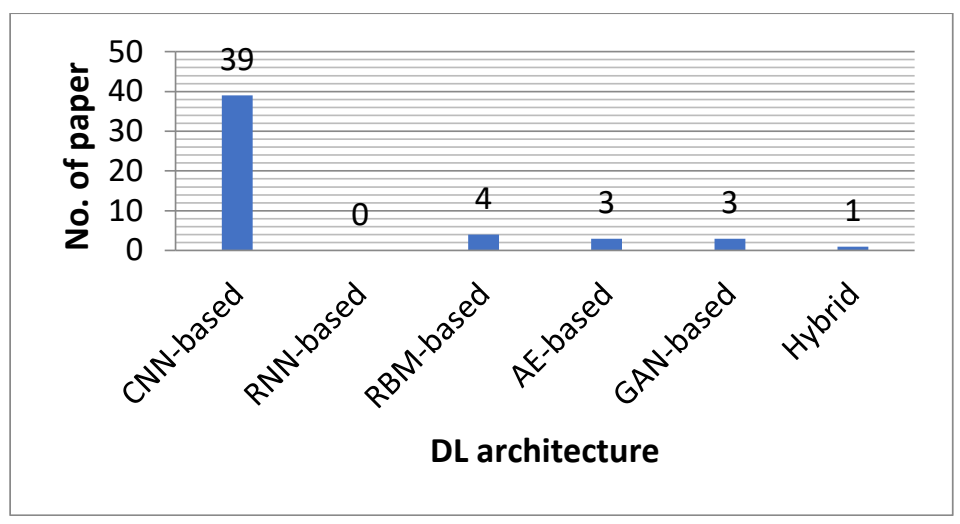

Figure 11. Number of papers based on DL architecture

\subsection{Task for fingerprint biometrics}

Based on the reviewed papers, it can be observed that there are wide applications of DL to extract global features (such as Arch, loop, delta, and whorl) and local features (minutiae) and sweat pores (fine-detail features) for the purpose of fingerprint matching. Some studies used DL approach to enhance and preprocess fingerprint images for better quality and for improving AFRS performance. Few studies on GAN demonstrate the efficacy of DL in generating fingerprint images. Different application tasks of the proposed DL-based methods have been identified such as fingerprint classification [15, 67, 79-81, 97, 100, 101, 111, 112], fingerprint liveness detection $[65,74,75,77,106,110]$, fingerprint recognition and authentication [25, 26, 76], overlapped fingerprint separation [86], double-identity fingerprint detection [87], fingerprint ROI segmentation [88, 89], fingerprint alteration detection [94], fingerprint image enhancement [20, 96, 107, 108], latent fingerprint segmentation [83], latent fingerprint recognition [85], latent fingerprint enhancement [84], fingerprint indexing [90, 91], fingerprint pore matching [70, 99], partial fingerprint matching[92, 93], cancelable recognition system [95], fingerprint spoofing detection [6, 109], contactless to contact-based and 3D partial fingerprint images matching [104, 105], fingerprint minutiae extraction [71, 102, 103], fingerprint pore extraction [98], fingerprint generation, and presentation attack detection [113, 114], fingerprint recovery scheme [115], and fingerprint localization, and detection [116].

\subsection{Datasets}

There are various datasets developed for evaluating and testing fingerprint recognition models. Some of the public available datasets include:

1) LivDet Competitions databases: These are public available databases that compare biometric liveness detection methodologies using a standardized testing protocol and large quantities of spoof and live samples. The LivDet competitions that have been hosted include LivDet 2009, 2011, 2013, 
2015, 2017, 2019, and 2021. However, LivDet 2021 was not used by any of the included study to the best of the author's knowledge.

2) FVC Fingerprint Databases: These databases are publicly provided and widely used for fingerprint evaluation. The FVC datasets contain two types of dataset Set A and Set B. Each set consists of 4 subsets, DB1, DB2, DB3 (real) and DB4 (synthetic). FVC 2002 consists of three fingerprint datasets (DB1, DB2, and DB3) collected using the optical sensor except for DB3 which was collected using capacitive sensor. The FVC 2004 also contains DB1, DB2, DB3 collected using the optical sensor except for DB3 which was collected using thermal sweeping sensor. The DB4 was introduced more deliberate noise, e.g. skin distortions, skin moisture, and rotation. FCV 2000 was the first FCV database to use four different sensors to capture fingerprint that produced four different databases (DB1, DB2, DB3 and DB4).

3) PolyU High-resolution Fingerprint Database: This is a public dataset provided by the Hong Kong Polytechnic University. The dataset contains two databases (denoted as DBI and DBII) of high resolution fingerprint images containing 1480 images captured from 148 fingers.

4) Contactless 2D to Contact-based 2D Fingerprint Images Database: This is a public dataset provided by the Hong Kong Polytechnic University. It contains two-session database from more than 300 clients using contactless and contact-based traditional sensors, consists of 1800 2D contactless fingerprint images and corresponding 1800 2D contact-based fingerprints [119]

5) 3D Fingerprint Images Database: This is a public dataset provided by the Hong Kong Polytechnic University. This database contains new two-session 2D/3D fingerprints database acquiring from 300 different clients consisting of 2760 different 3D fingerprints and they are reconstructed from 5520 colored 2D fingerprints [120].

6) NIST Fingerprint Dataset: This is a public fingerprint databases. NIST SD27 consists of 258 latent fingerprints and corresponding reference fingerprints [121]. NIST special database 4 contains 8- bit gray scale images of randomly selected fingerprints[122].

Based on this study, it can be deduced that some datasets are freely available while some are private. The freely available datasets were created based on standards and published on the internet to enable free access for researchers and for cross evaluation. The private datasets are datasets acquired by the authors due to non-availability of the type of data required for the unique nature of the research.

\subsection{Modelling Deep Learning Architecture}

This section presents discussions on the training architectures used in the proposed models as reviewed in this paper. After the acquisition of fingerprint images from the various devices and methods, the training of the data involves basically three 
steps, the pre-processing, feature extraction and classification/detection/matching. Figure 12 depicts the conventional training architecture of biometric data.

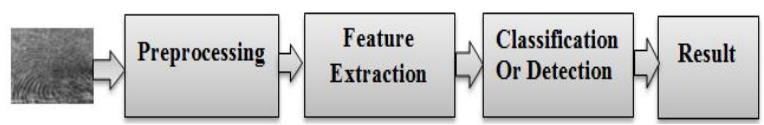

Figure 12. Conventional Training architecture

There are different combinations of DL with other traditional methods that were identified in the literature. There are some training approaches that a deep network was used for feature extraction and then fed into a shallow technique for classification described in section 5.4.1. On the other hand, some training architectures used shallow technique for feature extraction and then fed the features into a deep network for classification as discussed in section 5.4.2. However, there are some approaches that used end-to-end training, where input data (raw fingerprint images) are fed into a deep network for automatic data classification, described in detail in section 5.4.3.

RQ3: What are the roles of $D L$ in the training architectures employed in the proposed models?

Research question 3 seeks to find out the roles of DL in the training architectures in the proposed models.

\section{1) Traditional Method as Extractor and Deep network as Classifier}

In this training architecture, the DL methods were fed with the features extracted by the traditional methods, and classified the data based on the expected results. The architecture of DL as a classifier is graphically illustrated in Figure 13.

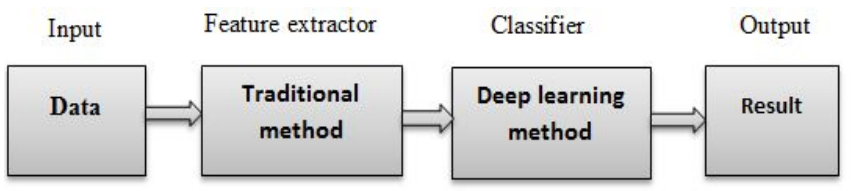

Figure 13. DL as classifier and traditional method as feature extractor

For instance, [15] proposed a fingerprint classification using Deep CNN classifier method which was fed with texture features extracted using histogram equalization, Gabor enhancement and fingerprint thinning. A study by [92] proposed a method for partial fingerprint matching. The method starts by aligning two fingerprints in a coarsely fashion using an algorithm based on phase-only correlation. The overlapped region was extracted. Lastly, a deep CNN with spatial pyramid pooling was used to verify whether these two fingerprint regions belong to the same finger. These approaches are employed to improve classification accuracy by transforming raw data into feature data which has better discriminated characteristics.

\section{2) Deep network as Feature Extractor and Traditional Method as Classifier}

In this training architecture, traditional methods were used to classify features extracted using DL. Figure 14 illustrate this architecture. 


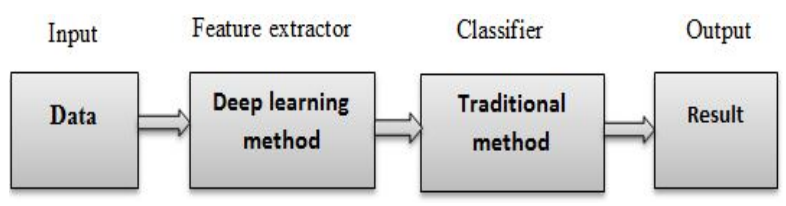

Figure 14. DL as feature extractor and traditional method as classifier

For instance, [77] proposed a study that utilized CNN with random weights as feature extractor and was compared with the classical LBP method. The extracted features from these methods were fed into the SVM classifier for learning. A study conducted by [65] for fingerprint liveness detection applied a modified classical $\mathrm{CNN}$ as a feature extractor. The extracted features were passed into machine learning classifiers (K-Nearest Neighbors, Logistic Regression and Naïve- Bayes classifier) for fingerprint liveness detection. These approaches take advantage of the DL robustness to extract more discriminative features from the image data for classification and aimed to avoid the cumbersome labeling of dataset using hand-crafted methods by employing DL to train the dataset in an unsupervised learning manner.

3) Deep network in end-to-end learning

There are training architectures that utilized a DL network both for data extraction and classification/authentication, where fingerprint images are fed into the network for automatic classification or matching. Figure 15 illustrates this architecture.

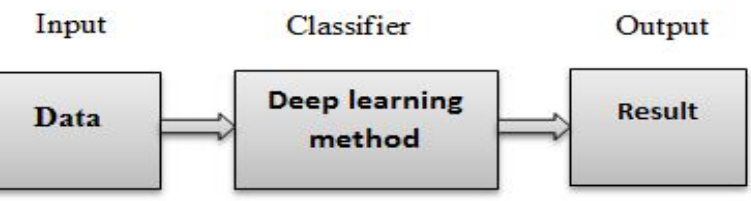

Figure 15. DL in end-to-end learning

For instance, a study conducted by [75] utilized pre-trained CNN based networks (CNN-VGG, CNN-Alexnet) and CNN-with random weights to detect live fingerprint images in an end-to-end learning. The study proposed by [86] introduces end-to-end separation of overlapped fingerprints using DL called FinSNet to automatically separate overlapped fingerprint for matching. In order to avoid the burden of hand-crafted feature extraction process, [110] applied stacked AE to automatically extract fingerprint features for real-time fingerprint liveness detection. Five DL models such as classic CNN, Alexnet, VGG-16, Yolo-v2 and Resnet-50 were used to automatically classify specific fingerprint information. Fingerprint classification method was performed by [80] using pre-trained CNN based networks (VGGF and VGG-S) to automatically classifies it. Methods that used DL in an end-to-end approached aimed at reducing the burden associated with hand-crafted feature extraction and for convenience. In these models, the stress of manual extraction of features is removed and no expertise is required to label the fingerprint images [106, 110].

\section{Open Challenges and Prospect for Future Research}

The DL based fingerprint algorithms and techniques are considered the best approach producing state-of-the-art results better than the conventional machine 
learning algorithms, fuzzy logic, latent, image enhancement, holistic, and template matching as discussed in [19]. There are wide applications of DL to analyze fingerprint images for various applications tasks with proven performances better than the traditional methods. However, there is still a room for improvement. Fingerprint recognition can be challenging, basically because of large intra-class variations caused by displacement, small inter-class variations, and strong noise in the fingerprint patterns, nonlinear distortion, variable pressure, skin condition, and more. These challenges would continue to be open research problems [3, 80, 81]. Based on this study it is observed that most of the proposed models utilized only four classes of fingerprint (left loop, right loop, arch, and whorl). However, the use of more than four classes of fingerprint would be helpful for research in human personality's diversity. A study conducted by [67] demonstrated the suitability and stability of a CNN based method (FCTP-Net) in identifying six-category fingerprint. This research area may be investigated more to promote research in the diversity of human personalities.

Although DL models are robust and produce state-of-the-art performances, but they are still susceptible to security challenges/attacks such as adversarial attacks, spoofing attacks, and presentation attacks [6, 109, 114, 123, 124]. For security reason, fingerprint biometric systems don't store fingerprint features as they are but save them as template which is considered relatively safe. However, research has shown that the fingerprint templates which are considered safe are no longer safe [115]. A DL based study by [115] obtained over $90 \%$ of presentation attack success rate when FMR was $0.1 \%$ using skeleton image recovered from the leaked fingerprint template. Although minutiae based methods have consistently proven effective in biometric research [19], however, vulnerable features such as fingerprint type feature, orientation map, and skeleton image in terms of security should be avoided when developing fingerprint biometric systems [115]. Also, designing security measures in biometric systems while at the same time maintaining the recognition accuracy of the systems is still being explored[29]

This study was restricted to uni-modal biometric (fingerprint biometric), however, studies have been proposed to take advantage of the strengths of the various biometric features through fusion of two or more biometrics to build a more accurate and secured identification also known as multimodal biometric systems [125-127]. However, the need of biometric data and challenges of reusability of biometric modules and unified frameworks has been a concerned for multimodal biometric systems [127].

DL models are data hungry architectures; they produce better performance with larger dataset. As observed in this study, most studies used limited number of dataset while some would have to create (acquire) dataset to train or test their models. Limited number of datasets may affect the generalizability and performance of the DL models. A study by [94], found it difficult to identify type of alteration due to lack of publicly available datasets that contain obfuscation and distortion of fingerprints. Transfer learning and data augmentation have been used when there was limited data to train the proposed models [25, 70, 75, 77, 79, 119]. More so, studies based on GAN to enhance generation of fingerprint images which may be helpful to demonstrate presentation attack detection have been proposed [113, 114]. Due to the proliferation of mobile devices, there have been mobile fingerprint scanners used to conveniently capture fingerprint. However, the problem with small finger area of mobile fingerprint scanner, inadvertent impressions, low quality of 
ridge impressions, and significant non-linear distortion continue to presents a challenge in partial fingerprint features [93].

The challenge in elastic deformations is inherent on images acquired from the contact-based fingerprint sensors. The deformation of contact-based fingerprint makes it challenging to achieve accurate minutiae extraction and matching using conventional methods. Contactless fingerprint identification systems have been proposed in the literature to mitigate the challenges of contact-based fingerprint extraction and matching[104, 119]. However, 3D contactless imaging requires relatively higher cooperation from users during the contactless 3D imaging, as a result contactless 3D imaging often results in partial 3D fingerprints [105]. The inculcation of 3D images and the integration of 2D and 3D biometric recognition techniques in biometric systems are considered a future potential in fingerprint biometric towards a more reliable and accurate fingerprint systems [7].

Based on this study, DL roles were observed in three ways; as feature extractor, classifier or end-to-end learning. A study to employ these three training architectures to train a model and evaluate the model using the same experimental settings and dataset may be helpful in understanding the advantages and disadvantages of the roles of DL in the training architecture.

\section{Conclusions}

This paper presented systematic literature review of 50 primary studies based on the applications of DL models in fingerprint biometric system. There are various analyses performed at various stages of AFRS with the aim to enhance the quality of fingerprint images and to improve AFRS performance. This study revealed evidences of the application of various schemes based on DL to model fingerprints images which includes fingerprint classification, fingerprint liveness detection, overlapped fingerprint separation, double-identity fingerprint detection, fingerprint ROI segmentation, fingerprint alteration detection, fingerprint image enhancement, latent fingerprint segmentation, latent fingerprint recognition, latent fingerprint enhancement, fingerprint indexing, fingerprint pore matching, partial fingerprint matching, cancelable recognition system, fingerprint spoofing detection, contactless to contact-based and 3D partial Fingerprint Images matching, fingerprint minutiae extraction, fingerprint pore extraction, fingerprint generation and presentation attack detection, fingerprint recovery scheme, fingerprint localization and detection. Among the DL algorithms, the CNN based models seems to be saturated for fingerprint biometric research and it would continue to remain relevant due to the inherent nature and robustness of CNN in image processing. The study also reported the role of DL in the training architecture of the proposed models. It was found that DL was used as a feature extractor, classifier or as an end-to-end learning model. However, there will be need to evaluate the advantages and disadvantages of these training architectures for the choice of a better training architecture.

Although DL have produced state-of-the-art performance in fingerprint biometric systems, yet, there is still great room for improvement such as improving classification accuracy using more number of fingerprint classes for recognition of human personality diversity, tackling the problem of large intra-class variations, small inter-class variations, and strong noise in the fingerprint patterns, creating new and unavailable datasets to make it accessible for cross evaluation, creating larger and more challenging databases, investigating more on 3D fingerprint biometric technology while incorporating security and privacy issues. 


\section{Conflicts of Interest}

The authors declare that they have no known competing financial interests or personal relationships that could have appeared to influence the work reported in this paper.

\section{References}

[1] Chollet, F.: 'Deep Learning mit Python und Keras: Das Praxis-Handbuch vom Entwickler der Keras-Bibliothek’ (MITP-Verlags GmbH \& Co. KG, 2018. 2018)

[2] Faust, O., Hagiwara, Y., Hong, T.J., Lih, O.S., and Acharya, U.R.: 'Deep learning for healthcare applications based on physiological signals: A review', Computer methods and programs in biomedicine, 2018, 161, pp. 1-13

[3] Sundararajan, K., and Woodard, D.L.: 'Deep learning for biometrics: A survey’, ACM Computing Surveys (CSUR), 2018, 51, (3), pp. 1-34

[4] Voulodimos, A., Doulamis, N., Doulamis, A., and Protopapadakis, E.: 'Deep learning for computer vision: A brief review', Computational intelligence and neuroscience, 2018, 2018

[5] Win, K.N., Li, K., Chen, J., Viger, P.F., and Li, K.: 'Fingerprint classification and identification algorithms for criminal investigation: A survey', Future Generation Computer Systems, 2020, 110, pp. 758-771

[6] Arora, S., and Bhatia, M.S.: 'Fingerprint spoofing detection to improve customer security in mobile financial applications using deep learning', Arabian journal for science and engineering, 2019, pp. 1-17

[7] Dargan, S., and Kumar, M.: 'A comprehensive survey on the biometric recognition systems based on physiological and behavioral modalities', Expert Systems with Applications, 2020, 143, pp. 113114

[8] Maltoni, D., Maio, D., Jain, A.K., and Prabhakar, S.: 'Handbook of fingerprint recognition’ (Springer Science \& Business Media, 2009. 2009)

[9] Jain, A.K., Prabhakar, S., and Pankanti, S.: 'On the similarity of identical twin fingerprints’, Pattern Recognition, 2002, 35, (11), pp. 2653-2663

[10] Bansal, R., Sehgal, P., and Bedi, P.: 'Minutiae extraction from fingerprint images-a review', IJCSI International Journal of Computer Science 2011, 8, (5), pp. 74-85

[11] Bowyer, K.W., Hollingsworth, K., and Flynn, P.J.: 'Image understanding for iris biometrics: A survey', Computer vision and image understanding, 2008, 110, (2), pp. 281-307

[12] Sarkar, I., Alisherov, F., Kim, T.-h., and Bhattacharyya, D.: 'Palm vein authentication system: a review', International Journal of Control and Automation, 2010, 3, (1)

[13] Monrose, F., and Rubin, A.D.: 'Keystroke dynamics as a biometric for authentication', Future Generation computer systems, 2000, 16, (4), pp. 351-359

[14] Hafemann, L.G., Sabourin, R., and Oliveira, L.S.: 'Offline handwritten signature verification-Literature review’, in Editor (Ed.)^(Eds.): 'Book Offline handwritten signature verification-Literature review’ (IEEE, 2017, edn.), pp. 1-8

[15] Pandya, B., Cosma, G., Alani, A.A., Taherkhani, A., Bharadi, V., and McGinnity, T.: 'Fingerprint classification using a deep convolutional neural network', in Editor (Ed.)^(Eds.): 'Book Fingerprint classification using a deep convolutional neural network’ (IEEE, 2018, edn.), pp. 86-91 
[16] Bote-Curiel, L., Munoz-Romero, S., Gerrero-Curieses, A., and Rojo-Álvarez, J.L.: 'Deep learning and big data in healthcare: A double review for critical beginners’, Applied Sciences, 2019, 9, (11), pp. 2331

[17] Park, U., Tong, Y., and Jain, A.K.: 'Age-invariant face recognition', IEEE transactions on pattern analysis and machine intelligence, 2010, 32, (5), pp. 947-954

[18] Guo, Y., Lei, Y., Liu, L., Wang, Y., Bennamoun, M., and Sohel, F.: 'EI3D: Expression-invariant 3D face recognition based on feature and shape matching', Pattern Recognition Letters, 2016, 83, pp. 403-412

[19] Ali, S.F., Khan, M.A., and Aslam, A.S.: 'Fingerprint matching, spoof and liveness detection: classification and literature review', Frontiers of Computer Science, 2021, 15, (1), pp. 1-18

[20] Wong, W.J., and Lai, S.-H.: 'Multi-task CNN for restoring corrupted fingerprint images’, Pattern Recognition, 2020, 101, pp. 107203

[21] Maheswari, S.U., and Chandra, E.: 'A review study on fingerprint classification algorithm used for fingerprint identification and recognition', IJCST, 2012, 3, (1), pp. 739-745

[22] Borra, S.R., Reddy, G.J., and Reddy, E.S.: 'A broad survey on fingerprint recognition systems', in Editor (Ed.)^(Eds.): 'Book A broad survey on fingerprint recognition systems' (IEEE, 2016, edn.), pp. 1428-1434

[23] Irum, S., Afzal, H., and Mahmud, S.: 'How to Build an Automated Fingerprint Identification System', in Editor (Ed.)^(Eds.): 'Book How to Build an Automated Fingerprint Identification System’ (IEEE, 2013, edn.), pp. 40-47

[24] Pathan, A.S.U., Thakur, K.K., Chakraborty, A., and Kabir, M.H.: 'Fingerprint Authentication Security: An Improved 2-Step Authentication Method with Flexibility', International Journal of Scientific \& Engineering Research, 2019, 10, (1)

[25] Minaee, S., Azimi, E., and Abdolrashidi, A.: 'Fingernet: Pushing the limits of fingerprint recognition using convolutional neural network', arXiv preprint arXiv:1907.12956, 2019

[26] Shuping, N., and Feng, W.: 'The research on fingerprint recognition algorithm fused with deep learning', in Editor (Ed.)^(Eds.): 'Book The research on fingerprint recognition algorithm fused with deep learning’ (IEEE, 2020, edn.), pp. 1044-1047

[27] Minaee, S., Abdolrashidi, A., Su, H., Bennamoun, M., and Zhang, D.: 'Biometrics recognition using deep learning: A survey', arXiv preprint arXiv:1912.00271, 2019

[28] Kiefer, R., and Patel, A.: 'A Comprehensive Survey on Fingerprint Liveness Detection Algorithms by Database and Scanner Model', Advances in Security, Networks, and Internet of Things, 2021, pp. 39-51

[29] Yang, W., Wang, S., Hu, J., Zheng, G., and Valli, C.: 'Security and accuracy of fingerprint-based biometrics: A review', Symmetry, 2019, 11, (2), pp. 141

[30] Militello, C., Rundo, L., Vitabile, S., and Conti, V.: 'Fingerprint Classification Based on Deep Learning Approaches: Experimental Findings and Comparisons', Symmetry, 2021, 13, (5), pp. 750

[31] Mehraj, H., and Mir, A.H.: 'A Survey of Biometric Recognition Using Deep Learning', 2020

[32] Ezhilmaran, D., and Adhiyaman, M.: 'A review study on fingerprint image enhancement techniques', International Journal of Computer Science \& Engineering Technology (IJCSET) ISSN, 2014, pp. 2229-3345

[33] Nguyen, G., Dlugolinsky, S., Bobák, M., Tran, V., García, Á.L., Heredia, I., Malík, P., and Hluchý, L.: 'Machine Learning and Deep Learning frameworks and 
libraries for large-scale data mining: a survey', Artificial Intelligence Review, 2019, 52, (1), pp. 77-124

[34] Alom, M.Z., Taha, T.M., Yakopcic, C., Westberg, S., Sidike, P., Nasrin, M.S., Hasan, M., Van Essen, B.C., Awwal, A.A., and Asari, V.K.: 'A state-of-the-art survey on deep learning theory and architectures', Electronics, 2019, 8, (3), pp. 292

[35] He, Z.: 'Deep Learning in Image Classification: A Survey Report', in Editor (Ed.)^(Eds.): 'Book Deep Learning in Image Classification: A Survey Report' (IEEE, 2020, edn.), pp. 174-177

[36] Roger, V., Farinas, J., and Pinquier, J.: 'Deep neural networks for automatic speech processing: a survey from large corpora to limited data', arXiv preprint arXiv:2003.04241, 2020

[37] Kumar, A., Verma, S., and Mangla, H.: 'A survey of deep learning techniques in speech recognition', in Editor (Ed.) ${ }^{\wedge}$ (Eds.): 'Book A survey of deep learning techniques in speech recognition’ (IEEE, 2018, edn.), pp. 179-185

[38] Jiao, L., Zhang, F., Liu, F., Yang, S., Li, L., Feng, Z., and Qu, R.: 'A survey of deep learning-based object detection', IEEE access, 2019, 7, pp. 128837-128868

[39] Alam, M., Samad, M.D., Vidyaratne, L., Glandon, A., and Iftekharuddin, K.M.: 'Survey on deep neural networks in speech and vision systems', Neurocomputing, 2020, 417, pp. 302-321

[40] Hubel, D.H., and Wiesel, T.N.: 'Receptive fields, binocular interaction and functional architecture in the cat's visual cortex', The Journal of physiology, 1962, 160, (1), pp. 106

[41] Shrestha, A., and Mahmood, A.: 'Review of deep learning algorithms and architectures’, IEEE Access, 2019, 7, pp. 53040-53065

[42] Yamashita, R., Nishio, M., Do, R.K.G., and Togashi, K.: 'Convolutional neural networks: an overview and application in radiology', Insights into imaging, 2018, 9, (4), pp. 611-629

[43] Pourbabaee, B., Roshtkhari, M.J., and Khorasani, K.: 'Deep convolutional neural networks and learning ECG features for screening paroxysmal atrial fibrillation patients', IEEE Transactions on Systems, Man, and Cybernetics: Systems, 2018, 48, (12), pp. 2095-2104

[44] Shi, H., Qin, C., Xiao, D., Zhao, L., and Liu, C.: 'Automated heartbeat classification based on deep neural network with multiple input layers', Knowledge-Based Systems, 2020, 188, pp. 105036

[45] Schmidhuber, J.: 'Deep learning in neural networks: An overview', Neural networks, 2015, 61, pp. 85-117

[46] Sornam, M., Muthusubash, K., and Vanitha, V.: 'A survey on image classification and activity recognition using deep convolutional neural network architecture', in Editor (Ed.)^(Eds.): 'Book A survey on image classification and activity recognition using deep convolutional neural network architecture' (IEEE, 2017, edn.), pp. 121-126

[47] Zeiler, M.D., and Fergus, R.: 'Visualizing and understanding convolutional networks', in Editor $(\text { Ed. })^{\wedge}($ Eds.): 'Book Visualizing and understanding convolutional networks’ (Springer, 2014, edn.), pp. 818-833

[48] He, K., Zhang, X., Ren, S., and Sun, J.: 'Deep residual learning for image recognition', in Editor (Ed.) $\wedge^{\wedge}$ Eds.): ‘Book Deep residual learning for image recognition' (2016, edn.), pp. 770-778

[49] Simonyan, K., and Zisserman, A.: 'Very deep convolutional networks for large-scale image recognition’, arXiv preprint arXiv:1409.1556, 2014 
[50] Iandola, F.N., Han, S., Moskewicz, M.W., Ashraf, K., Dally, W.J., and Keutzer, K.: 'SqueezeNet: AlexNet-level accuracy with 50x fewer parameters and< 0.5 MB model size', arXiv preprint arXiv:1602.07360, 2016

[51] Szegedy, C., Liu, W., Jia, Y., Sermanet, P., Reed, S., Anguelov, D., Erhan, D., Vanhoucke, V., and Rabinovich, A.: 'Going deeper with convolutions', in Editor (Ed.)^(Eds.): ‘Book Going deeper with convolutions' (2015, edn.), pp. 1-9

[52] Khan, A., Sohail, A., Zahoora, U., and Qureshi, A.S.: 'A survey of the recent architectures of deep convolutional neural networks', Artificial Intelligence Review, 2020, 53, (8), pp. 5455-5516

[53] Phung, V.H., and Rhee, E.J.: 'A deep learning approach for classification of cloud image patches on small datasets', Journal of information and communication convergence engineering, 2018, 16, (3), pp. 173-178

[54] Smolensky, P.: 'Information processing in dynamical systems: Foundations of harmony theory', in Editor (Ed.)^(Eds.): 'Book Information processing in dynamical systems: Foundations of harmony theory' (Colorado Univ at Boulder Dept of Computer Science, 1986, edn.), pp.

[55] Sengupta, S., Basak, S., Saikia, P., Paul, S., Tsalavoutis, V., Atiah, F., Ravi, V., and Peters, A.: 'A review of deep learning with special emphasis on architectures, applications and recent trends', Knowledge-Based Systems, 2020, pp. 105596

[56] Montúfar, G.: 'Restricted boltzmann machines: Introduction and review', in Editor (Ed.) $)^{\wedge}$ (Eds.): 'Book Restricted boltzmann machines: Introduction and review' (Springer, 2016, edn.), pp. 75-115

[57] Chen, X.-M., Wu, C.-X., Wu, Y., Xiong, N.-X., Han, R., Ju, B.-B., and Zhang, S.: 'Design and analysis for early warning of rotor UAV based on data-driven DBN', Electronics, 2019, 8, (11), pp. 1350

[58] LeCun, Y., Bottou, L., Bengio, Y., and Haffner, P.: 'Gradient-based learning applied to document recognition', Proceedings of the IEEE, 1998, 86, (11), pp. 2278-2324

[59] Bank, D., Koenigstein, N., and Giryes, R.: 'Autoencoders', arXiv preprint arXiv:2003.05991, 2020

[60] Goodfellow, I., Pouget-Abadie, J., Mirza, M., Xu, B., Warde-Farley, D., Ozair, S., Courville, A., and Bengio, Y.: 'Generative adversarial nets', in Editor (Ed.)^(Eds.): ‘Book Generative adversarial nets’ (2014, edn.), pp. 2672-2680

[61] Hayes, J., Melis, L., Danezis, G., and Cristofaro, E.: 'LOGAN: Evaluating Information Leakage of Generative Models Using Generative Adversarial Networks', arXiv preprint arXiv:1705.07663, 2017

[62] Jabbar, A., Li, X., and Omar, B.: 'A survey on generative adversarial networks: Variants, applications, and training', arXiv preprint arXiv:2006.05132, 2020

[63] Mason, J., Dave, R., Chatterjee, P., Graham-Allen, I., Esterline, A., and Roy, K.: 'An Investigation of Biometric Authentication in the Healthcare Environment', Array, 2020, 8, pp. 100042

[64] Ali, M.M., Mahale, V.H., Yannawar, P., and Gaikwad, A.: 'Overview of fingerprint recognition system', in Editor (Ed.)^(Eds.): 'Book Overview of fingerprint recognition system' (IEEE, 2016, edn.), pp. 1334-1338

[65] TK, A.K., Vinayakumar, R., Sowmya, V., and Soman, K.: 'Convolutional

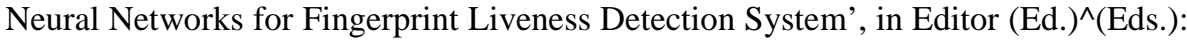
'Book Convolutional Neural Networks for Fingerprint Liveness Detection System' (IEEE, 2019, edn.), pp. 243-246

[66] Henry, E.R.: 'Classification and uses of finger prints' (HM Stationery Office, printed by Darling and son, Limited, 1913. 1913) 
[67] Wu, F., Zhu, J., and Guo, X.: 'Fingerprint pattern identification and classification approach based on convolutional neural networks', Neural Computing and Applications, 2019, pp. 1-10

[68] Peralta, D., Triguero, I., García, S., Saeys, Y., Benitez, J.M., and Herrera, F.: 'Distributed incremental fingerprint identification with reduced database penetration rate using a hierarchical classification based on feature fusion and selection', Knowledge-Based Systems, 2017, 126, pp. 91-103

[69] Abdullah, H.A.: 'Fingerprint identification system using neural networks', Al-Nahrain Journal for Engineering Sciences, 2012, 15, (2), pp. 234-244

[70] Liu, Y., Zhou, B., Han, C., Guo, T., and Qin, J.: 'A novel method based on deep learning for aligned fingerprints matching’, Applied Intelligence, 2020, 50, (2), pp. 397-416

[71] Tang, Y., Gao, F., Feng, J., and Liu, Y.: 'FingerNet: An unified deep network for fingerprint minutiae extraction', in Editor (Ed.)^(Eds.): 'Book FingerNet: An unified deep network for fingerprint minutiae extraction’ (IEEE, 2017, edn.), pp. 108-116

[72] Kitchenham, B., and Charters, S.: 'Guidelines for performing systematic literature reviews in software engineering version 2.3', Engineering, 2007, 45, (4ve), pp. 1051

[73] Xiao, Y., and Watson, M.: 'Guidance on conducting a systematic literature review', Journal of Planning Education and Research, 2019, 39, (1), pp. 93-112

[74] Park, E., Cui, X., Kim, W., and Kim, H.: 'End-to-end fingerprints liveness detection using convolutional networks with gram module', arXiv preprint arXiv:1803.07830, 2018

[75] Nogueira, R.F., de Alencar Lotufo, R., and Machado, R.C.: 'Fingerprint liveness detection using convolutional neural networks', IEEE transactions on information forensics and security, 2016, 11, (6), pp. 1206-1213

[76] Amruth, Y., Gopinatha, B., Gowtham, M., Kiran, M., and Harshalatha, Y.: 'Fingerprint and Signature Authentication System using CNN', in Editor (Ed.) $)^{(E d s .): ~ ' B o o k ~ F i n g e r p r i n t ~ a n d ~ S i g n a t u r e ~ A u t h e n t i c a t i o n ~ S y s t e m ~ u s i n g ~ C N N ' ~}$ (IEEE, 2020, edn.), pp. 1-4

[77] Nogueira, R.F., de Alencar Lotufo, R., and Machado, R.C.: 'Evaluating software-based fingerprint liveness detection using convolutional networks and local binary patterns’, in Editor (Ed.)^(Eds.): 'Book Evaluating software-based fingerprint liveness detection using convolutional networks and local binary patterns' (IEEE, 2014, edn.), pp. 22-29

[78] Sheena, S., and Mathew, S.: 'Fingerprint Classification with reduced penetration rate: Using Convolutional Neural Network and DeepLearning', in Editor (Ed.)^(Eds.): 'Book Fingerprint Classification with reduced penetration rate: Using Convolutional Neural Network and DeepLearning' (IEEE, 2018, edn.), pp. 2141-2144

[79] Tertychnyi, P., Ozcinar, C., and Anbarjafari, G.: 'Low-quality fingerprint classification using deep neural network', IET Biometrics, 2018, 7, (6), pp. 550-556 [80] Michelsanti, D., Ene, A.-D., Guichi, Y., Stef, R., Nasrollahi, K., and Moeslund, T.B.: 'Fast fingerprint classification with deep neural networks', in Editor (Ed.)^(Eds.): 'Book Fast fingerprint classification with deep neural networks' (SCITEPRESS Digital Library, 2017, edn.), pp. 202-209

[81] Ge, S., Bai, C., Liu, Y., Liu, Y., and Zhao, T.: 'Deep and discriminative feature learning for fingerprint classification', in Editor (Ed.)^(Eds.): 'Book Deep and 
discriminative feature learning for fingerprint classification' (IEEE, 2017, edn.), pp. 1942-1946

[82] Zhang, N., Zang, Y., Jia, X., Yang, X., and Tian, J.: 'Define a fingerprint orientation field pattern', in Editor (Ed.)^(Eds.): 'Book Define a fingerprint orientation field pattern' (IEEE, 2016, edn.), pp. 937-942

[83] Zhu, Y., Yin, X., Jia, X., and Hu, J.: 'Latent fingerprint segmentation based on convolutional neural networks', in Editor (Ed.)^(Eds.): 'Book Latent fingerprint segmentation based on convolutional neural networks' (IEEE, 2017, edn.), pp. 1-6

[84] Li, J., Feng, J., and Kuo, C.-C.J.: 'Deep convolutional neural network for latent fingerprint enhancement’, Signal Processing: Image Communication, 2018, 60, pp. 52-63

[85] Cao, K., and Jain, A.K.: 'Automated latent fingerprint recognition', IEEE transactions on pattern analysis and machine intelligence, 2018, 41, (4), pp. 788-800 [86] Yoo, D., Cho, J., Lee, J., Chae, M., Lee, B., and Lee, B.: 'FinSNet: End-to-End Separation of Overlapped Fingerprints Using Deep Learning', IEEE Access, 2020, 8, pp. 209020-209029

[87] Goel, I., Puhan, N.B., and Mandal, B.: 'Deep convolutional neural network for double-identity fingerprint detection', IEEE Sensors Letters, 2020, 4, (5), pp. 1-4

[88] Stojanović, B., Marques, O., Nešković, A., and Puzović, S.: 'Fingerprint ROI segmentation based on deep learning', in Editor (Ed.)^(Eds.): 'Book Fingerprint ROI segmentation based on deep learning’ (IEEE, 2016, edn.), pp. 1-4

[89] Joshi, I., Kothari, R., Utkarsh, A., Kurmi, V.K., Dantcheva, A., Roy, S.D., and Kalra, P.K.: 'Explainable fingerprint roi segmentation using Monte Carlo dropout', in Editor (Ed.)^(Eds.): 'Book Explainable fingerprint roi segmentation using Monte Carlo dropout' (IEEE Computer Society, 2021, edn.), pp. 60-69

[90] Bai, C.-c., Wang, W.-q., Zhao, T., Wang, R.-x., and Li, M.-q.: 'Deep learning compact binary codes for fingerprint indexing', Frontiers of Information Technology \& Electronic Engineering, 2018, 19, (9), pp. 1112-1123

[91] Song, D., Tang, Y., and Feng, J.: 'Aggregating minutia-centred deep convolutional features for fingerprint indexing', Pattern Recognition, 2019, 88, pp. 397-408

[92] Qin, J., Tang, S., Han, C., and Guo, T.: 'Partial fingerprint matching via phase-only correlation and deep convolutional neural network', in Editor (Ed.)^(Eds.): 'Book Partial fingerprint matching via phase-only correlation and deep convolutional neural network' (Springer, 2017, edn.), pp. 602-611

[93] Zeng, F., Hu, S., and Xiao, K.: 'Research on partial fingerprint recognition algorithm based on deep learning’, Neural Computing and Applications, 2019, 31, (9), pp. 4789-4798

[94] Shehu, Y.I., Ruiz-Garcia, A., Palade, V., and James, A.: 'Detection of fingerprint alterations using deep convolutional neural networks', in Editor (Ed.)^(Eds.): 'Book Detection of fingerprint alterations using deep convolutional neural networks' (Springer, 2018, edn.), pp. 51-60

[95] Abdellatef, E., Omran, E.M., Soliman, R.F., Ismail, N.A., Abd Elrahman, S.E.S., Ismail, K.N., Rihan, M., Abd El-Samie, F.E., and Eisa, A.A.: 'Fusion of deep-learned and hand-crafted features for cancelable recognition systems', Soft Computing, 2020, 24, (20), pp. 15189-15208

[96] Karabulut, D., Tertychnyi, P., Arslan, H.S., Ozcinar, C., Nasrollahi, K., Valls, J., Vilaseca, J., Moeslund, T.B., and Anbarjafari, G.: 'Cycle-consistent generative adversarial neural networks based low quality fingerprint enhancement', Multimedia Tools and Applications, 2020, 79, (25), pp. 18569-18589 
[97] Rim, B., Kim, J., and Hong, M.: 'Fingerprint classification using deep learning approach', Multimedia Tools and Applications, 2020, pp. 1-17

[98] Su, H.-R., Chen, K.-Y., Wong, W.J., and Lai, S.-H.: 'A deep learning approach towards pore extraction for high-resolution fingerprint recognition', in Editor (Ed.)^(Eds.): 'Book A deep learning approach towards pore extraction for high-resolution fingerprint recognition' (IEEE, 2017, edn.), pp. 2057-2061

[99] Liu, F., Zhao, Y., Liu, G., and Shen, L.: 'Fingerprint pore matching using deep features', Pattern Recognition, 2020, 102, pp. 107208

[100] Peralta, D., Triguero, I., García, S., Saeys, Y., Benitez, J.M., and Herrera, F.: 'On the use of convolutional neural networks for robust classification of multiple fingerprint captures’, International Journal of Intelligent Systems, 2018, 33, (1), pp. 213-230

[101] Jeon, W.-S., and Rhee, S.-Y.: 'Fingerprint pattern classification using convolution neural network', International Journal of Fuzzy Logic and Intelligent Systems, 2017, 17, (3), pp. 170-176

[102] Jiang, L., Zhao, T., Bai, C., Yong, A., and Wu, M.: 'A direct fingerprint minutiae extraction approach based on convolutional neural networks', in Editor (Ed.)^(Eds.): 'Book A direct fingerprint minutiae extraction approach based on convolutional neural networks' (IEEE, 2016, edn.), pp. 571-578

[103] Darlow, L.N., and Rosman, B.: 'Fingerprint minutiae extraction using deep learning', in Editor $(E d .)^{\wedge}(E d s$.$) : ‘Book Fingerprint minutiae extraction using$ deep learning' (IEEE, 2017, edn.), pp. 22-30

[104] Lin, C., and Kumar, A.: 'Multi-siamese networks to accurately match contactless to contact-based fingerprint images', in Editor (Ed.)^(Eds.): 'Book Multi-siamese networks to accurately match contactless to contact-based fingerprint images' (IEEE, 2017, edn.), pp. 277-285

[105] Lin, C., and Kumar, A.: 'Contactless and partial 3D fingerprint recognition using multi-view deep representation', Pattern Recognition, 2018, 83, pp. 314-327

[106] Kim, S., Park, B., Song, B.S., and Yang, S.: 'IDeep belief network based statistical feature learning for fingerprint liveness detection’, Pattern Recognition Letters, 2016, 77, pp. 58-65

[107] Sahasrabudhe, M., and Namboodiri, A.M.: 'Learning fingerprint orientation fields using continuous restricted Boltzmann machines', in Editor (Ed.) $)^{\wedge}$ (Eds.): 'Book Learning fingerprint orientation fields using continuous restricted Boltzmann machines’ (IEEE, 2013, edn.), pp. 351-355

[108] Sahasrabudhe, M., and Namboodiri, A.M.: 'Fingerprint enhancement using unsupervised hierarchical feature learning', in Editor (Ed.)^(Eds.): 'Book Fingerprint enhancement using unsupervised hierarchical feature learning' (2014, edn.), pp. 1-8

[109] Uliyan, D.M., Sadeghi, S., and Jalab, H.A.: 'Anti-spoofing method for fingerprint recognition using patch based deep learning machine’, Engineering Science and Technology, an International Journal, 2020, 23, (2), pp. 264-273

[110] Yuan, C., Chen, X., Yu, P., Meng, R., Cheng, W., Wu, Q.J., and Sun, X.: 'Semi-supervised stacked autoencoder-based deep hierarchical semantic feature for real-time fingerprint liveness detection', Journal of Real-Time Image Processing, 2020, 17, (1), pp. 55-71

[111] Wang, R., Han, C., Wu, Y., and Guo, T.: 'Fingerprint classification based on depth neural network', arXiv preprint arXiv:1409.5188, 2014 
[112] Wang, R., Han, C., and Guo, T.: 'A novel fingerprint classification method based on deep learning', in Editor (Ed.)^(Eds.): ‘Book A novel fingerprint classification method based on deep learning’ (IEEE, 2016, edn.), pp. 931-936

[113] Minaee, S., and Abdolrashidi, A.: 'Finger-gan: Generating realistic fingerprint images using connectivity imposed gan', arXiv preprint arXiv:1812.10482, 2018

[114] Kim, H., Cui, X., Kim, M.-G., and Nguyen, T.H.B.: 'Fingerprint generation and presentation attack detection using deep neural networks', in Editor (Ed.)^(Eds.): 'Book Fingerprint generation and presentation attack detection using deep neural networks' (IEEE, 2019, edn.), pp. 375-378

[115] Lee, S., Jang, S.-W., Kim, D., Hahn, H., and Kim, G.-Y.: 'A Novel Fingerprint Recovery Scheme using Deep Neural Network-based Learning', Multimedia Tools and Applications, 2020, pp. 1-15

[116] Tabassi, E., Chugh, T., Deb, D., and Jain, A.K.: 'Altered fingerprints: Detection and localization', in Editor (Ed.)^(Eds.): 'Book Altered fingerprints: Detection and localization' (IEEE, 2018, edn.), pp. 1-9

[117] Rumelhart, D.E., Hinton, G.E., and Williams, R.J.: 'Learning representations by back-propagating errors', nature, 1986, 323, (6088), pp. 533-536

[118] Salehinejad, H., Sankar, S., Barfett, J., Colak, E., and Valaee, S.: 'Recent advances in recurrent neural networks', arXiv preprint arXiv:1801.01078, 2017

[119] Lin, C., and Kumar, A.: 'Matching contactless and contact-based conventional fingerprint images for biometrics identification', IEEE Transactions on Image Processing, 2018, 27, (4), pp. 2008-2021

[120] Lin, C., and Kumar, A.: 'Tetrahedron based fast 3D fingerprint identification using colored LEDs illumination', IEEE transactions on pattern analysis and machine intelligence, 2017, 40, (12), pp. 3022-3033

[121] Garris, D.M., and McCabe, R.: 'NIST Special Database 27 Fingerprint Minutiae From Latent and Matching Tenprint Images, Special Publication (NIST SP), National Institute of Standards and Technology, Gaithersburg, MD, ' 2000

[122] Watson, C.I., and Wilson, C.L.: 'NIST special database 4', Fingerprint Database, National Institute of Standards and Technology, 1992, 17, (77), pp. 5

[123] Ross, A.A., Shah, J., and Jain, A.K.: 'Toward reconstructing fingerprints from minutiae points', in Editor (Ed.)^(Eds.): 'Book Toward reconstructing fingerprints from minutiae points' (International Society for Optics and Photonics, 2005, edn.), pp. 68-80

[124] Fei, J., Xia, Z., Yu, P., and Xiao, F.: 'Adversarial attacks on fingerprint liveness detection', EURASIP Journal on Image and Video Processing, 2020, 2020, (1), pp. 1-11

[125] Sarhan, S., Alhassan, S., and Elmougy, S.: 'Multimodal biometric systems: a comparative study', Arabian Journal for Science and Engineering, 2017, 42, (2), pp. 443-457

[126] Ghayoumi, M.: 'A review of multimodal biometric systems: Fusion methods and their applications', in Editor (Ed.)^(Eds.): 'Book A review of multimodal biometric systems: Fusion methods and their applications’ (IEEE, 2015, edn.), pp. 131-136

[127] Ouch, R., Garcia-Zapirain, B., and Yampolskiy, R.: 'Multimodal biometrie systems: A systematic review’, in Editor (Ed.)^(Eds.): 'Book Multimodal biometrie systems: A systematic review’ (IEEE, 2017, edn.), pp. 439-444 